\title{
Hochschild cohomology versus the Jacobian ring and the Torelli theorem for cubic fourfolds
}

\author{
Daniel Huybrechts and Jørgen Vold Rennemo
}

\begin{abstract}
The Jacobian ring $J(X)$ of a smooth hypersurface $X \subset \mathbb{P}^{n+1}$ determines the isomorphism type of $X$. This has been used by Donagi and others to prove the generic global Torelli theorem for hypersurfaces in many cases. However, in Voisin's original proof (and, in fact, in all other proofs) of the global Torelli theorem for smooth cubic fourfolds $X \subset \mathbb{P}^{5}$, the Jacobian ring does not intervene. In this paper, we present a proof of the global Torelli theorem for cubic fourfolds that relies on the Jacobian ring and the (derived) global Torelli theorem for K3 surfaces. It emphasizes, once again, the close and still mysterious relation between K3 surfaces and smooth cubic fourfolds.

More generally, for a variant of Hochschild cohomology $\mathrm{HH}^{*}\left(\mathcal{A}_{X},(1)\right)$ of Kuznetsov's category $\mathcal{A}_{X}$ (together with the degree-shift functor (1)) associated with an arbitrary smooth hypersurface $X \subset \mathbb{P}^{n+1}$ of degree $d \leqslant n+2$, we construct a graded-ring homomorphism $J(X) \longrightarrow \mathrm{HH}^{*}\left(\mathcal{A}_{X},(1)\right)$, which is shown to be bijective whenever $\mathcal{A}_{X}$ is a Calabi-Yau category.
\end{abstract}

\section{Introduction}

The derived category $\mathrm{D}^{\mathrm{b}}(X)$ of a smooth hypersurface $X \subset \mathbb{P}^{n+1}$ of degree $d \neq n+2$ determines the hypersurface $X$ uniquely. However, a certain full triangulated subcategory $\mathcal{A}_{X} \subset \mathrm{D}^{\mathrm{b}}(X)$ introduced by Kuznetsov in [Kuz10] turns out to be a subtler and more interesting derived invariant of $X$. The case of cubic fourfolds $X \subset \mathbb{P}^{5}$ has been studied intensively; cf. [AT14, Huy17, Kuz10, MS12]. As observed by Kuznetsov, in this case $\mathcal{A}_{X}$ behaves in many respects like the derived category $\mathrm{D}^{\mathrm{b}}(S)$ of a K3 surface $S$. In particular, its Hochschild cohomology $\mathrm{HH}^{*}\left(\mathcal{A}_{X}\right)$ is known to be isomorphic to the Hochschild cohomology of a K3 surface.

The aim of the paper is twofold. We introduce a version of Hochschild cohomology of $\mathcal{A}_{X}$ for smooth hypersurfaces $X \subset \mathbb{P}^{n+1}$, denoted by $\mathrm{HH}^{*}\left(\mathcal{A}_{X},(1)\right)$, and explain its relation to the Jacobian ring $J(X)$. The main result here is the following (cf. Corollary 3.7).

Theorem 1.1. For any smooth hypersurface $X \subset \mathbb{P}^{n+1}$ of degree $d \leqslant(n+2) / 2$, there exists a natural surjective homomorphism of graded rings $\pi: J(X) \longrightarrow \mathrm{HH}^{*}\left(\mathcal{A}_{X},(1)\right)$, which is an isomorphism if $n+2$ is divisible by $d$.

Received 27 April 2017, accepted in final form 21 September 2017.

2010 Mathematics Subject Classification 14J28, 14F05, 14J35.

Keywords: K3 surfaces, cubic fourfolds, Torelli theorem, Jacobian ring, Hochschild cohomology, derived category. This journal is (C) Foundation Compositio Mathematica 2019. This article is distributed with Open Access under the terms of the Creative Commons Attribution Non-Commercial License, which permits non-commercial reuse, distribution, and reproduction in any medium, provided that the original work is properly cited. For commercial re-use, please contact the Foundation Compositio Mathematica.

The first author was supported by the SFB/TR 45 'Periods, Moduli Spaces and Arithmetic of Algebraic Varieties' of the DFG (German Research Foundation). 


\section{HoCHSCHILD COHOMOLOGY VERSUS THE JACOBIAN RING}

Note that the numerical assumption $d \mid(n+2)$ is exactly the one that according to Kuznetsov [Kuz18] ensures that $\mathcal{A}_{X}$ is a Calabi-Yau category. The theorem can be viewed as a graded version of Dyckerhoff's description of the Hochschild cohomology of the category of (ungraded) matrix factorizations [Dyc11].

The category $\mathcal{A}_{X}$ is naturally endowed with an auto-equivalence (1), the degree-shift functor, which can be described either as the line bundle twist $\otimes \mathcal{O}_{X}(1)$ followed by the projection onto $\mathcal{A}_{X}$, see Section 2, or as the degree shift by interpreting $\mathcal{A}_{X}$ as the category of graded matrix factorizations; see Section 6. A purely categorical consequence of Theorem 1.1 is the following (see Corollary 3.10 for details).

Corollary 1.2. Two smooth hypersurfaces $X, X^{\prime} \subset \mathbb{P}^{n+1}$ of degree $d \mid(n+2)$ with $1<d<n+2$ are isomorphic if and only if there exists a Fourier-Mukai equivalence $\left(\mathcal{A}_{X},(1)\right) \simeq\left(\mathcal{A}_{X^{\prime}},(1)\right)$.

Using this, we can provide a new proof of the global Torelli theorem for cubic fourfolds based on the (derived) global Torelli theorem for K3 surfaces.

Theorem 1.3. Two smooth complex cubic fourfolds $X, X^{\prime} \subset \mathbb{P}^{5}$ are isomorphic if and only if there exists a Hodge isometry $H^{4}(X, \mathbb{Z})_{\mathrm{pr}} \simeq H^{4}\left(X^{\prime}, \mathbb{Z}\right)_{\mathrm{pr}}$.

The proof presented here passes via an isomorphism of Jacobian rings $J(X) \simeq J\left(X^{\prime}\right)$ and so is closer in spirit to Donagi's generic Torelli theorems for hypersurfaces [Don83] than to the original by Voisin [Voi86]. We certainly make no claim that our arguments are any easier or more natural than the existing ones. But it is certainly interesting to see that the result can be deduced directly from the global Torelli theorem for K3 surfaces, demonstrating once more the fascinating and mysterious link between cubic fourfolds and K3 surfaces.

In the rest of the introduction, we provide more background for both parts of the paper.

1.1. Global Torelli theorem. The classical Torelli theorem asserts that two smooth complex projective curves $C$ and $C^{\prime}$ are isomorphic if and only if there exists a Hodge isometry $H^{1}(C, \mathbb{Z}) \simeq$ $H^{1}\left(C^{\prime}, \mathbb{Z}\right)$. The Hodge structure is the usual Hodge structure of weight one, and the pairing is provided by the intersection product. ${ }^{1}$

Due to results of Pjateckiı̌-Šapiro and Šafarevič [PS71] and Burns and Rapoport [BR75], a similar result holds true for K3 surfaces. More precisely, two K3 surfaces $S$ and $S^{\prime}$ are isomorphic if and only if there exists a Hodge isometry $H^{2}(S, \mathbb{Z}) \simeq H^{2}\left(S^{\prime}, \mathbb{Z}\right)$. The Hodge structure is of weight two, and the pairing is again given by the intersection product. ${ }^{2}$

More recently, Verbitsky [Ver13] proved a version of the global Torelli theorem for compact hyperkähler manifolds, higher-dimensional versions of K3 surfaces, which shows that two such manifolds $Y$ and $Y^{\prime}$ are birational if and only if there exists a Hodge isometry $H^{2}(Y, \mathbb{Z}) \simeq$ $H^{2}\left(Y^{\prime}, \mathbb{Z}\right)$ (with respect to the Beauville-Bogomolov pairing) which is a parallel transport operator; cf. [Huy12, Mar11].

Classically, a similar question has been asked for smooth hypersurfaces. Concretely, are two smooth hypersurfaces $X, X^{\prime} \subset \mathbb{P}^{n+1}$ isomorphic if and only if there exists a Hodge isometry between their primitive middle cohomology $H^{n}(X, \mathbb{Z})_{\mathrm{pr}} \simeq H^{n}\left(X^{\prime}, \mathbb{Z}\right)_{\mathrm{pr}}$ ? The question has been

\footnotetext{
${ }^{1}$ If the condition on the compatibility with the pairing is dropped, then the Jacobians of $C$ and $C^{\prime}$ are still isomorphic, $J(C) \simeq J\left(C^{\prime}\right)$, as unpolarized abelian varieties and, in particular, $\left[S^{n} C\right]=\left[S^{n} C^{\prime}\right]$ for $n>2 g-2$ in the Grothendieck ring of varieties $K_{0}$ (Var).

${ }^{2}$ If the intersection product is ignored, then the Hodge conjecture still predicts the isomorphism to be induced by an algebraic class on the product, but its concrete geometric meaning is unclear.
} 


\section{Huybrechts And J. V. Rennemo}

addressed and answered in most cases by Donagi [Don83]. Combined with the later work by Donagi-Green [DG84] and Cox-Green [CG90], his results can be stated as follows.

Theorem 1.4 (Donagi, Cox, Green). The global Torelli theorem holds for generic hypersurfaces $X, X^{\prime} \subset \mathbb{P}^{n+1}$ of degree $d$ except possibly in the following cases:

$$
\text { (i) }(d, n)=(3,2), \quad \text { (ii) }(d, n)=(4,4 m), \quad \text { and } \quad \text { (iii) } d \mid(n+2) \text {. }
$$

Note that the third exception corresponds to the situation considered in Theorem 1.1 and ensures that $\mathcal{A}_{X}$ is a Calabi-Yau category [Kuz18].

Also note that in the exceptions (i)-(iii), $X$ is either a Fano or a Calabi-Yau variety. The latter is the case $d=n+2$ in exception (iii). In the Calabi-Yau situation, the global Torelli theorem is known to hold (and not only generically) for the cases $(d, n)=(3,1)$ (elliptic curves), $(d, n)=(4,2)$ (quartic K3 surfaces), and $(d, n)=(5,3)$ (quintic threefolds). The first two are either trivial or special cases of the global Torelli theorem for K3 surfaces. The case of quintic threefolds is much harder and has been settled by Voisin in [Voi99].

In the Fano situation, the global Torelli theorem really fails for $(d, n)=(3,2)$, for the Hodge structure of a cubic surface $X \subset \mathbb{P}^{3}$ is of type $(1,1)$ and thus cannot distinguish between nonisomorphic cubic surfaces. So, the first interesting case is that of cubic fourfolds $X \subset \mathbb{P}^{5}$. Again, the global Torelli theorem is known to hold for those, a result due to Voisin [Voi86, Voi08]. Her proof eventually relies on the global Torelli theorem for K3 surfaces of degree two (for which a direct proof was given by Shah [Sha80]). Another proof for cubic fourfolds, not drawing upon K3 surfaces, was given by Looijenga in [Loo09] and yet another more recent one by Charles [Cha12] uses Verbitsky's global Torelli theorem applied to the hyperkähler fourfold provided by the Fano variety of lines $F(X)$.

Donagi's proof of the generic global Torelli theorem for hypersurfaces uses the period map to identify certain graded parts of the Jacobian rings of $X$ and $X^{\prime}$. Applying his symmetrizer lemma [Don83, Proposition 6.2], for which one has to exclude cases (i)-(iii), allows him to deduce from this a graded-ring isomorphism $J(X) \simeq J\left(X^{\prime}\right)$. A version of the Mather-Yau theorem then implies $X \simeq X^{\prime}$. The argument breaks down for the exceptional cases and, indeed, in the existing proofs of the global Torelli theorem for cubic fourfolds, the Jacobian ring makes no appearance.

The idea of our approach is to show that whenever there exists a Hodge isometry $H^{4}(X, \mathbb{Z})_{\mathrm{pr}} \simeq$ $H^{4}\left(X^{\prime}, \mathbb{Z}\right)_{\text {pr }}$ between two (very general) smooth cubic fourfolds, the K3 categories $\mathcal{A}_{X}$ and $\mathcal{A}_{X^{\prime}}$ are equivalent. This relies on the derived global Torelli theorem for K3 surfaces due to Orlov [Or197] and the result of Addington and Thomas [AT14] showing, in particular, that the set of cubics $X$ for which $\mathcal{A}_{X}$ is equivalent to the bounded derived category $\mathrm{D}^{\mathrm{b}}(S)$ of some K3 surface $S$ is dense. If an equivalence $\mathcal{A}_{X} \simeq \mathcal{A}_{X^{\prime}}$ in addition commutes with the natural autoequivalence (1) given by mapping an object $E$ to the projection of $E \otimes \mathcal{O}(1)$ (called the degreeshift functor), then $\mathrm{HH}^{*}\left(\mathcal{A}_{X},(1)\right) \simeq \mathrm{HH}^{*}\left(\mathcal{A}_{X^{\prime}},(1)\right)$ essentially by the definition of the Hochschild cohomology of $(\mathcal{A},(1))$. Theorem 1.1 then yields a graded-ring isomorphism $J(X) \simeq J\left(X^{\prime}\right)$ and, by the Mather-Yau theorem, an isomorphism $X \simeq X^{\prime}$. In order to reduce to the situation where the equivalence $\mathcal{A}_{X} \simeq \mathcal{A}_{X^{\prime}}$ indeed commutes with the degree-shift functor, one needs to argue that the set of cubics $X$ for which $\mathcal{A}_{X} \simeq \mathrm{D}^{\mathrm{b}}(S, \alpha)$ for some twisted K3 surface $(S, \alpha)$ without any spherical objects is dense in the moduli space; cf. [Huy17]. This suffices to conclude the compatibility with the degree-shift functor, since the results of [HMS08] show that the group of auto-equivalences of $\mathrm{D}^{\mathrm{b}}(S, \alpha)$ is essentially trivial. 


\section{HochSCHILD COHOMOLOGY VERSUS THE JACOBIAN RING}

1.2. Graded matrix factorizations. Kuznetsov's category $\mathcal{A}_{X}$ of a hypersurface $X \subset \mathbb{P}^{n+1}$ defined by an equation $f \in k\left[x_{0}, \ldots, x_{n+1}\right]$ has been shown to be equivalent to the category of graded matrix factorizations $\operatorname{MF}(f, \mathbb{Z})$; see [Orl09] and Section 6 for the definition and some facts. Although we do not make use of this equivalence, it served as a motivation for our approach. In particular, Dyckerhoff's description [Dyc11] of the Hochschild cohomology $\mathrm{HH}^{*}(\mathrm{MF}(f))$ of the category of ungraded matrix factorization as the Jacobian ring $J(X)$ got this project started.

More precisely, Dyckerhoff studies an isolated hypersurface singularity, that is, a regular local $k$-algebra $R$, and a non-unit $f \in R$ such that the quotient $R /(f)$ has an isolated singularity. He then shows that the Hochschild cohomology $\mathrm{HH}^{*}(\mathrm{MF}(f))$ (which is concentrated in even degree) of the differential graded category of $\mathbb{Z} / 2 \mathbb{Z}$-periodic matrix factorizations is isomorphic to the Jacobian ring $R /\left(\partial_{i} f\right)$; cf. [Dyc11, Corollary 6.5].

The naive original idea of our approach was to say that any equivalence $\mathcal{A}_{X} \simeq \mathcal{A}_{X^{\prime}}$, interpreted as an equivalence $\mathrm{MF}(f, \mathbb{Z}) \simeq \mathrm{MF}\left(f^{\prime}, \mathbb{Z}\right)$, that commutes with the degree-shift functor $(1)$ on both sides descends to an equivalence $\operatorname{MF}(f) \simeq \operatorname{MF}(f, \mathbb{Z}) /(1) \simeq \operatorname{MF}\left(f^{\prime}, \mathbb{Z}\right) /(1) \simeq \operatorname{MF}\left(f^{\prime}\right)$. The latter then induces a ring isomorphism $J(X) \simeq J\left(X^{\prime}\right)$.

There are, however, a number of problems that one has to address when using the equivalence $\mathcal{A}_{X} \simeq \operatorname{MF}(f, \mathbb{Z})$. First, this is an equivalence of triangulated categories. It comes with an enhancement, but in order to apply any graded version of [Dyc11], one would need to make sure that the enhancement for $\operatorname{MF}(f, \mathbb{Z})$ corresponds to the one used by Dyckerhoff. Also, the compatibility of the equivalence $\mathcal{A}_{X} \simeq \mathcal{A}_{X^{\prime}}$ with the degree-shift functor would need to be lifted to the enhancement. Second, the naive idea to pass from the category $\operatorname{MF}(f, \mathbb{Z})$ to the quotient $\operatorname{MF}(f)=\operatorname{MF}(f, \mathbb{Z}) /(1)$ needs to be spelled out and possibly be lifted to the enhancements. Third, the relation between the degree-shift functors $(k)$ for $\operatorname{MF}(f, \mathbb{Z})$ and the auto-equivalences of $\mathcal{A}_{X}$ is rather technical; see [BFK12].

So, we decided to work entirely on the derived side $\mathcal{A}_{X} \subset \mathrm{D}^{\mathrm{b}}(X)$ and adapted Kuznetsov's philosophy that viewing $\mathcal{A}_{X}$ as an admissible subcategory of $\mathrm{D}^{\mathrm{b}}(X)$ and working exclusively with Fourier-Mukai kernels replaces the choice of a dg-enhancement for $\mathcal{A}_{X}$.

Versions of Hochschild cohomology for categories of graded matrix factorizations have been introduced and studied in [BFK14], in which a relation to the Jacobian ring was also explained; see Section 6 for further comments.

\section{Kuznetsov's category $\mathcal{A}_{X}$ from the kernel perspective}

In this section, we first recall the definition of the category $\mathcal{A}_{X}$ for a smooth hypersurface $X \subset \mathbb{P}^{n+1}$ (over an arbitrary field of characteristic zero) of degree $d$ and state Kuznetsov's result saying that it is a Calabi-Yau category under suitable assumptions on $d$ and $n$. Then we revisit the auto-equivalence (1): $\mathcal{A}_{X} \stackrel{\sim}{\longrightarrow} \mathcal{A}_{X}, E \longmapsto i^{*}\left(E \otimes \mathcal{O}_{X}(1)\right)$ (the degree-shift functor) and Kuznetsov's central observation that the $d$-fold composition $(d)$ is the double shift [2]. As for our purposes it is important to understand this not only as an isomorphism of functors but as an isomorphism between their Fourier-Mukai kernels, we essentially re-prove his result in the kernel setting. This then allows us to factor the isomorphism $(d) \simeq[2]$ through the tangent bundle, see Lemma 2.19, which is crucial for proving the existence of the ring homomorphism from the Jacobian ring $J(X)$ to the Hochschild cohomology $\mathrm{HH}^{*}\left(\mathcal{A}_{X},(1)\right)$. 


\section{Huybrechts and J. V. Rennemo}

2.1. Let $X \subset \mathbb{P}:=\mathbb{P}^{n+1}$ be a smooth hypersurface of degree $d$, and let

$$
\mathcal{A}_{X}:=\left\langle\mathcal{O}_{X}, \ldots, \mathcal{O}_{X}(n+1-d)\right\rangle^{\perp} \subset \mathrm{D}^{\mathrm{b}}(X)
$$

be the full triangulated subcategory of all objects $E$ with $\operatorname{Hom}^{*}\left(\mathcal{O}_{X}(\ell), E\right)=0$ for all $\ell=$ $0, \ldots, n+1-d$. We will denote the image of $\mathcal{A}_{X}$ under the line bundle twist $E \longmapsto E \otimes \mathcal{O}_{X}(\ell)$ by $\mathcal{A}_{X}(\ell)$, which can also be described as $\left\langle\mathcal{O}_{X}(\ell), \ldots, \mathcal{O}_{X}(n+1-d+\ell)\right\rangle^{\perp}$.

By definition, the left orthogonal ${ }^{\perp} \mathcal{A}_{X}$ is the full triangulated subcategory spanned by the exceptional collection $\mathcal{O}_{X}, \ldots, \mathcal{O}_{X}(n+1-d)$. This yields a semi-orthogonal decomposition

$$
\mathrm{D}^{\mathrm{b}}(X)=\left\langle\mathcal{A}_{X}, \mathcal{O}_{X}, \ldots, \mathcal{O}_{X}(n+1-d)\right\rangle
$$

see [BK89, BO95]. In particular, the inclusion $i_{*}: \mathcal{A}_{X} \hookrightarrow \mathrm{D}^{\mathrm{b}}(X)$ admits right- and left-adjoint functors

$$
i^{!}, i^{*}: \mathrm{D}^{\mathrm{b}}(X) \rightarrow \mathcal{A}_{X}
$$

(also called right and left projections), so that there exist functorial isomorphisms

$$
\operatorname{Hom}_{\mathrm{D}^{\mathrm{b}}(X)}\left(i_{*} E, F\right) \simeq \operatorname{Hom}_{\mathcal{A}_{X}}\left(E, i^{!} F\right) \quad \text { and } \operatorname{Hom}_{\mathrm{D}^{\mathrm{b}}(X)}\left(F, i_{*} E\right) \simeq \operatorname{Hom}_{\mathcal{A}_{X}}\left(i^{*} F, E\right)
$$

for all $E \in \mathcal{A}_{X}$ and $F \in \mathrm{D}^{\mathrm{b}}(X)$. With this notation, any object $E \in \mathrm{D}^{\mathrm{b}}(X)$ sits in unique exact triangles

$$
E^{\prime} \rightarrow E \rightarrow i_{*} i^{*} E \text { and } i_{*} i^{!} E \rightarrow E \rightarrow E^{\prime \prime}
$$

with $E^{\prime} \in\left\langle\mathcal{O}_{X}, \ldots, \mathcal{O}_{X}(n+1-d)\right\rangle={ }^{\perp} \mathcal{A}_{X}$ and $E^{\prime \prime} \in\left\langle\mathcal{O}_{X}(d-n-2), \ldots, \mathcal{O}_{X}(-1)\right\rangle=\mathcal{A}_{X}^{\perp}$.

The category $\mathrm{D}^{\mathrm{b}}(X)$ is endowed with a Serre functor described by $S_{X}: E \longmapsto E \otimes \mathcal{O}_{X}(d-$ $n-2)[n]$, and a Serre functor on the admissible subcategory $\mathcal{A}_{X} \subset \mathrm{D}^{\mathrm{b}}(X)$ is then given by $S_{\mathcal{A}_{X}} \simeq i^{!} \circ S_{X} \circ i_{*}$. This isomorphism can also be read as a description of $i^{!}$as $S_{\mathcal{A}_{X}} \circ i^{*} \circ S_{X}^{-1}$.

The category $\mathcal{A}_{X}$ is called a Calabi-Yau category if $S_{\mathcal{A}_{X}}$ is isomorphic to a shift functor $E \longmapsto E[N]$, in which case $N$ is called its dimension. For instance, if $d=n+2$, then $X$ itself is a Calabi-Yau variety and, in particular, $\mathrm{D}^{\mathrm{b}}(X)$ is a Calabi-Yau category (of dimension $n$ ).

See Remark 2.18 for an argument proving the next result in the case of cubic fourfolds.

Theorem 2.1 (Kuznetsov [Kuz18, Theorem 3.5]). Assume $d \mid(n+2)$. Then $\mathcal{A}_{X}$ is a Calabi-Yau category of dimension $(n+2)(d-2) / d$.

Clearly, with $\mathcal{A}_{X}$ also all twists $\mathcal{A}_{X}(\ell)$ are Calabi-Yau categories (of the same dimension).

Remark 2.2. As shall be explained, it is no accident that the Jacobian ring

$$
J(X):=k\left[x_{0}, \ldots, x_{n+1}\right] /\left(\partial_{i} f\right)
$$

of the equation $f \in k\left[x_{0}, \ldots, x_{n+1}\right]_{d}$ defining the hypersurface $X \subset \mathbb{P}^{n+1}$ is of top degree $(n+$ $2)(d-2)$; see [Don83, Theorem 2.5].

EXAMPLE 2.3. The first interesting cases occur for $d=3$ and hypersurfaces $X \subset \mathbb{P}^{n+1}$ of dimension $n=4,7,10, \ldots$ In these cases, the Calabi-Yau categories $\mathcal{A}_{X}$ are of dimensions $N=$ $2,3,4, \ldots$, respectively. Besides the case of a quartic K3 surfaces $X \subset \mathbb{P}^{3}$, where $\mathcal{A}_{X}=\mathrm{D}^{\mathrm{b}}(X)$, the case of a cubic fourfold $X \subset \mathbb{P}^{5}$ is the only one that leads to a Calabi-Yau category of dimension two (namely a K3 category).

2.2. Kuznetsov [Kuz11] associates with any subcategory $\mathcal{B} \subset \mathrm{D}^{\mathrm{b}}(X)$, say full triangulated and closed under taking direct summands, a subcategory $\mathcal{B} \otimes \mathrm{D}^{\mathrm{b}}(X) \subset \mathrm{D}^{\mathrm{b}}(X \times X)$. By definition, it 


\section{HochSCHILD COHOMOLOGY VERSUS THE JACOBIAN RING}

is the smallest full triangulated subcategory closed under taking direct summands that contains all objects of the form $E \otimes F:=p_{1}^{*} E \otimes p_{2}^{*} F$ with $E \in \mathcal{B}$ and $F \in \mathrm{D}^{\mathrm{b}}(X)$. The subcategory $\mathrm{D}^{\mathrm{b}}(X) \otimes \mathcal{B}$ is defined similarly. Note that $\mathrm{D}^{\mathrm{b}}(X) \otimes \mathrm{D}^{\mathrm{b}}(X) \simeq \mathrm{D}^{\mathrm{b}}(X \times X)$. According to [Kuz11, Proposition 5.1], any semi-orthogonal decomposition $\mathrm{D}^{\mathrm{b}}(X)=\left\langle\mathcal{A}_{1}, \ldots, \mathcal{A}_{m}\right\rangle$ induces a semiorthogonal decomposition $\mathrm{D}^{\mathrm{b}}(X \times X)=\left\langle\mathcal{A}_{1} \otimes \mathrm{D}^{\mathrm{b}}(X), \ldots, \mathcal{A}_{m} \otimes \mathrm{D}^{\mathrm{b}}(X)\right\rangle$. Moreover, in this case (cf. [Kuz11, Proposition 5.2]),

$$
\mathcal{A}_{i} \otimes \mathrm{D}^{\mathrm{b}}(X)=\left\{E \in \mathrm{D}^{\mathrm{b}}(X \times X) \mid p_{1 *}\left(E \otimes p_{2}^{*} F\right) \in \mathcal{A}_{i} \text { for all } F \in \mathrm{D}^{\mathrm{b}}(X)\right\} .
$$

We shall also need the exterior product $\mathcal{B} \otimes \mathcal{B}^{\prime} \subset \mathrm{D}^{\mathrm{b}}(X \times X)$ of two categories $\mathcal{B}, \mathcal{B}^{\prime} \subset \mathrm{D}^{\mathrm{b}}(X)$. As introduced in [Kuz11, Section 5.5], this is the intersection of $\mathcal{B} \otimes \mathrm{D}^{\mathrm{b}}(X)$ and $\mathrm{D}^{\mathrm{b}}(X) \otimes \mathcal{B}^{\prime}$ in $\mathrm{D}^{\mathrm{b}}(X \times X){ }^{3}$ For two semi-orthogonal decompositions $\mathrm{D}^{\mathrm{b}}(X)=\left\langle\mathcal{A}_{1}, \ldots, \mathcal{A}_{m}\right\rangle$ and $\mathrm{D}^{\mathrm{b}}(X)=$ $\left\langle\mathcal{A}_{1}^{\prime}, \ldots, \mathcal{A}_{n}^{\prime}\right\rangle$, the products $\mathcal{A}_{i} \otimes \mathcal{A}_{j}^{\prime} \subset \mathrm{D}^{\mathrm{b}}(X \times X)$ are admissible subcategories and, in fact, describe a semi-orthogonal decomposition of $\mathrm{D}^{\mathrm{b}}(X \times X)$; see [Kuz11, Theorem 5.8]. The case of interest to us is the product

$$
\mathcal{A}_{X}(-(n+1-d)) \otimes \mathcal{A}_{X} \subset \mathrm{D}^{\mathrm{b}}(X \times X),
$$

which can alternatively be described as the subcategory right orthogonal to $\left\langle\mathcal{O}_{X}(-(n+1-\right.$ $\left.d)), \ldots, \mathcal{O}_{X}\right\rangle \otimes \mathrm{D}^{\mathrm{b}}(X)$ and $\mathrm{D}^{\mathrm{b}}(X) \otimes\left\langle\mathcal{O}_{X}, \ldots, \mathcal{O}_{X}(n+1-d)\right\rangle$. We shall denote the inclusion $(2.2)$ by $j_{*}$ and its right and left adjoints by

$$
j^{!}, j^{*}: \mathrm{D}^{\mathrm{b}}(X \times X) \rightarrow \mathcal{A}_{X}(-(n+1-d)) \otimes \mathcal{A}_{X} \subset \mathrm{D}^{\mathrm{b}}(X \times X) .
$$

As a consequence of Theorem 2.1, one finds the following.

Corollary 2.4. Assume $d \mid(n+2)$. Then for all $\ell$, the product $\mathcal{A}_{X}(\ell) \otimes \mathcal{A}_{X}$ is a Calabi-Yau category of dimension $2(n+2)(d-2) / d$.

Proof. Consider the left and right projections $j^{*}, j^{!}: \mathrm{D}^{\mathrm{b}}(X \times X) \rightarrow \mathcal{A}_{X}(-(n+1-d)) \otimes \mathcal{A}_{X}$ of the inclusion, which can be written as the compositions of left and right projections id $\otimes i^{*}$, id $\otimes$ $i^{!}: \mathrm{D}^{\mathrm{b}}(X \times X) \rightarrow \mathrm{D}^{\mathrm{b}}(X) \otimes \mathcal{A}_{X}$ and $i^{*} \otimes \mathrm{id}, i^{!} \otimes \mathrm{id}: \mathrm{D}^{\mathrm{b}}(X) \otimes \mathcal{A}_{X} \subset \mathrm{D}^{\mathrm{b}}(X \times X) \rightarrow \mathcal{A}_{X}(-(n+1-$ d)) $\otimes \mathrm{D}^{\mathrm{b}}(X)$, for all of which the Fourier-Mukai kernels (see below) are obtained by base change from the ones for $i^{*}$ and $i^{!}$. Now, together with the comparison $S \circ j^{*} \simeq j^{!} \circ S_{X \times X}$ of $j_{*}$ and $j^{!}$, which can also be read as a description of the Serre functor $S$ of $\mathcal{A}_{X}(-(n+1-d)) \otimes \mathcal{A}_{X}$, and the relation between $i^{*}$ and $i^{!}$obtained from $S_{\mathcal{A}_{X}} \simeq[(n+2)(d-2) / d]$ (see Theorem 2.1), this yields the assertion.

2.3. For $P \in \mathrm{D}^{\mathrm{b}}(X \times X)$, we denote by $\Phi_{P}: \mathrm{D}^{\mathrm{b}}(X) \rightarrow \mathrm{D}^{\mathrm{b}}(X)$ the Fourier-Mukai functor $E \longmapsto p_{2 *}\left(p_{1}^{*} E \otimes P\right)$. Applying Kuznetsov's arguments [Kuz11], one easily finds the following result.

Lemma 2.5. (i) The essential image of $\Phi_{P}$ is contained in $\mathcal{A}_{X} \subset \mathrm{D}^{\mathrm{b}}(X)$ if and only if $P \in$ $\mathrm{D}^{\mathrm{b}}(X) \otimes \mathcal{A}_{X}$.

(ii) There exists a factorization of $\Phi_{P}$ via the projection $i^{*}: \mathrm{D}^{\mathrm{b}}(X) \rightarrow \mathcal{A}_{X}$, that is, $\Phi_{P}=0$ on $\langle\mathcal{O}, \ldots, \mathcal{O}(n+1-d)\rangle$, if and only if $P \in \mathcal{A}_{X}(-(n+1-d)) \otimes \mathrm{D}^{\mathrm{b}}(X)$.

\footnotetext{
${ }^{3}$ The notation may suggest to define $\mathcal{B} \otimes \mathcal{B}^{\prime}$ as the smallest triangulated subcategory that is closed under taking direct summands and contains all objects of the form $E \otimes E^{\prime}$ with $E \in \mathcal{B}$ and $E^{\prime} \in \mathcal{B}^{\prime}$. But this a priori produces a smaller subcategory. However, if $\mathcal{B}$ and $\mathcal{B}^{\prime}$ are components of semi-orthogonal decompositions, this description is valid, as was explained to us by Alex Perry.
} 


\section{Huybrechts and J. V. Rennemo}

Proof. The first assertion follows from (2.1). For the second, use that $\Phi_{P}\left(\mathcal{O}_{X}(\ell)\right)=0$ implies $\operatorname{Hom}_{X \times X}\left(p_{1}^{*} \mathcal{O}_{X}(-\ell) \otimes p_{2}^{*} E, P\right) \simeq \operatorname{Hom}_{X}\left(E, p_{2 *}\left(p_{1}^{*} \mathcal{O}_{X}(\ell) \otimes P\right)\right)=0$ for all $E \in \mathrm{D}^{\mathrm{b}}(X)$. Hence, if $\Phi_{P}=0$ on $\left\langle\mathcal{O}_{X}, \ldots, \mathcal{O}_{X}(n+1-d)\right\rangle$, then $P \in\left\langle\mathcal{O}_{X}(-(n+1-d)), \ldots, \mathcal{O}_{X}\right\rangle^{\perp} \otimes \mathrm{D}^{\mathrm{b}}(X)=$ $\mathcal{A}_{X}(-(n+1-d)) \otimes \mathrm{D}^{\mathrm{b}}(X)$.

Corollary 2.6. There exists a factorization

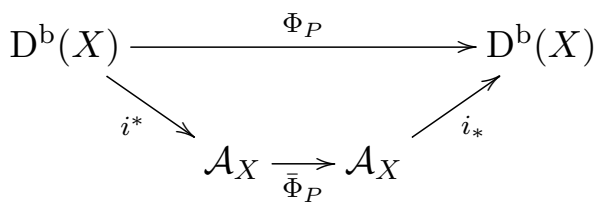

if and only if $P \in \mathcal{A}_{X}(-(n+1-d)) \otimes \mathcal{A}_{X}$.

Definition 2.7. A functor $\Phi: \mathcal{A}_{X} \rightarrow \mathcal{A}_{X}$ is called a Fourier-Mukai functor if it is isomorphic to a functor of the form $\bar{\Phi}_{P}$ with $P \in \mathcal{A}_{X}(-(n+1-d)) \otimes \mathcal{A}_{X}$ as above.

For any $P \in \mathrm{D}^{\mathrm{b}}(X \times X)$, one can consider $j^{*} P \in \mathcal{A}_{X}(-(n+1-d)) \otimes \mathcal{A}_{X}$, which then defines a Fourier-Mukai functor $\bar{\Phi}_{j^{*} P}: \mathcal{A}_{X} \rightarrow \mathcal{A}_{X}$.

Remark 2.8. The notion of a Fourier-Mukai functor $\bar{\Phi}_{P}: \mathcal{A}_{X} \rightarrow \mathcal{A}_{X^{\prime}}$ between the Calabi-Yau categories of two different hypersurfaces is defined similarly. In this case, the kernel is contained in $\mathcal{A}_{X}(-(n+1-d)) \otimes \mathcal{A}_{X^{\prime}}$.

EXAmple 2.9. In the following, we shall denote by $\Delta=\Delta_{X} \subset X \times X$ the diagonal (and also the diagonal embedding).

(i) Clearly, $\Phi_{\mathcal{O}_{\Delta}} \simeq$ id, but also $\bar{\Phi}_{j^{*} \mathcal{O}_{\Delta}} \simeq$ id. We shall use the shorthand

$$
P_{0}:=j^{*} \mathcal{O}_{\Delta} .
$$

As observed in [Kuz09, Proposition 3.8] (see also Lemma 2.13), $P_{0}$ can also be obtained as the image of $\mathcal{O}_{\Delta}$ under the left projection onto $\mathrm{D}^{\mathrm{b}}(X) \otimes \mathcal{A}_{X}$ or onto $\mathcal{A}_{X}(-(n+1-d)) \otimes \mathrm{D}^{\mathrm{b}}(X)$. In other words, the cone of the adjunction $\mathcal{O}_{\Delta} \rightarrow j^{*} \mathcal{O}_{\Delta} \simeq P_{0}$ is contained in the intersection of $\left\langle\mathcal{O}_{X}(-(n+1-d)), \ldots, \mathcal{O}_{X}\right\rangle \otimes \mathrm{D}^{\mathrm{b}}(X)$ and $\mathrm{D}^{\mathrm{b}}(X) \otimes\left\langle\mathcal{O}_{X}, \ldots, \mathcal{O}_{X}(n+1-d)\right\rangle$. Indeed, the left projection image of $\mathcal{O}_{\Delta}$ in $\mathrm{D}^{\mathrm{b}}(X) \otimes \mathcal{A}_{X}$, considered as a Fourier-Mukai kernel, is automatically trivial on $\left\langle\mathcal{O}_{X}(-(n+1-d)), \ldots, \mathcal{O}_{X}\right\rangle$; then use Lemma 2.5. Similarly, its image in $\mathcal{A}_{X}(-(n+$ $1-d)) \otimes \mathrm{D}^{\mathrm{b}}(X)$, considered as a Fourier-Mukai kernel, already takes values in $\mathcal{A}_{X}$.

(ii) The functor $\Phi_{\mathcal{O}_{\Delta}(\ell)}: \mathrm{D}^{\mathrm{b}}(X) \rightarrow \mathrm{D}^{\mathrm{b}}(X)$ is the line bundle twist $E \longmapsto E \otimes \mathcal{O}_{X}(\ell)$. We are particularly interested in the case $\ell=1$ and denote the projection of the corresponding kernel by

$$
P_{1}:=j^{*} \mathcal{O}_{\Delta}(1) .
$$

The induced functor was introduced by Kuznetsov in [Kuz04, Section 4]:

$$
(1):=\bar{\Phi}_{P_{1}}: \mathcal{A}_{X} \rightarrow \mathcal{A}_{X}, E \longmapsto i^{*}\left(E \otimes \mathcal{O}_{X}(1)\right) \text {. }
$$

We call (1) the degree-shift functor, which is motivated by interpreting $\mathcal{A}_{X}$ as a category of graded matrix factorizations; see Section 6.

Remark 2.10. Recall that for two objects $P, Q \in \mathrm{D}^{\mathrm{b}}(X \times X)$, the convolution $P \circ Q \in \mathrm{D}^{\mathrm{b}}(X \times X)$ is defined as $p_{13 *}\left(p_{12}^{*} P \otimes p_{23}^{*} Q\right)$. Then $\Phi_{P \circ Q} \simeq \Phi_{Q} \circ \Phi_{P}: \mathrm{D}^{\mathrm{b}}(X) \rightarrow \mathrm{D}^{\mathrm{b}}(X)$; see [Huy06, Chapter 5]. Note that if $P$ and $Q$ are contained in $\mathcal{A}_{X}(-(n+1-d)) \otimes \mathcal{A}_{X}$, then so is the convolution $P \circ Q$. 


\section{HochSCHILD COHOMOLOGY VERSUS THE JACOBIAN RING}

As an application, we rephrase the observation in Example 2.9(i) and write $P_{0}$ as the convolution

$$
P_{0} \simeq\left[\mathcal{O}(-m, m) \rightarrow \mathcal{O}_{\Delta}\right] \circ\left[\mathcal{O}(-m+1, m-1) \rightarrow \mathcal{O}_{\Delta}\right] \circ \cdots \circ\left[\mathcal{O} \longrightarrow \mathcal{O}_{\Delta}\right]
$$

where $\mathcal{O}(a, b):=\mathcal{O}(a) \otimes \mathcal{O}(b)$ and $m:=n+1-d$.

Remark 2.11. It is not difficult to show that for any $P \in \mathrm{D}^{\mathrm{b}}(X \times X)$, the projection $j^{*} P \in$ $\mathcal{A}_{X}(-(n+1-d)) \otimes \mathcal{A}_{X}$ is isomorphic to the right-left convolution with $P_{0}$; that is,

$$
j^{*} P \simeq P_{0} \circ P \circ P_{0} .
$$

Indeed, for every kernel $P$, there exists an exact triangle $P^{\prime} \rightarrow P \longrightarrow j^{*} P$ with $P^{\prime}$ contained in the category spanned by $\mathrm{D}^{\mathrm{b}}(X) \otimes\left\langle\mathcal{O}_{X}, \ldots, \mathcal{O}_{X}(n+1-d)\right\rangle$ and $\left\langle\mathcal{O}_{X}(-(n+1-d)), \ldots, \mathcal{O}_{X}\right\rangle \otimes \mathrm{D}^{\mathrm{b}}(X)$. Convoluting $P^{\prime}$ with $P_{0}$ (or any object in $\left.\mathcal{A}_{X}(-(n+1-d)) \otimes \mathcal{A}_{X}\right)$ from both sides is trivial and, therefore, $P_{0} \circ P \circ P_{0} \simeq P_{0} \circ j^{*} P \circ P_{0}$. Similarly, by the arguments in Example 2.9(i), convoluting $\mathcal{O}_{\Delta}^{\prime} \longrightarrow \mathcal{O}_{\Delta} \longrightarrow P_{0}$ with $j^{*} P$ from the left and with $j^{*} P \circ P_{0}$ from the right yields isomorphisms $j^{*} P \simeq j^{*} P \circ P_{0} \simeq P_{0} \circ j^{*} P \circ P_{0}$.

As a special case, we record that

$$
P_{1} \simeq P_{0} \circ \mathcal{O}_{\Delta}(1) \circ P_{0} \simeq P_{0} \circ \mathcal{O}_{\Delta}(1) \circ\left[\mathcal{O} \rightarrow \mathcal{O}_{\Delta}\right],
$$

where for the second isomorphism we use (2.4) and $\mathcal{A}_{X}(1) \subset\left\langle\mathcal{O}_{X}(1), \ldots, \mathcal{O}_{X}(m)\right\rangle^{\perp}$.

The $\ell$-fold convolution of $P_{1}$ with itself yields

$$
P_{\ell}:=P_{1}^{\circ \ell}:=P_{1} \circ \cdots \circ P_{1} \in \mathcal{A}_{X}(-(n+1-d)) \otimes \mathcal{A}_{X},
$$

whose induced Fourier-Mukai functor $\Phi_{P_{\ell}}$ is isomorphic to $(\ell):=(1)^{\ell}: \mathcal{A}_{X} \rightarrow \mathcal{A}_{X}$.

Remark 2.12. In fact, Kuznetsov shows [Kuz18, Corollary 3.18] that the functor (1) (which is his $\left.\mathrm{O}\right|_{\mathcal{A}_{X}}$ ) is an equivalence and clearly so are all $(\ell)$. Alternatively, this can be deduced from Corollary 2.16; see Remark 2.17.

Note that, in general, neither is the kernel $P_{\ell}$ isomorphic to $j^{*} \mathcal{O}_{\Delta}(\ell)$ nor is the functor $(\ell)$ isomorphic to $\bar{\Phi}_{j^{*} \mathcal{O}_{\Delta}(\ell)}$. However, for $\ell=0, \ldots, d$, Kuznetsov establishes this isomorphism [Kuz18, Proposition 3.17], which is made explicit by the following kernel version, crucial for our purposes. See Corollary 2.16 for a characterization of all $P_{\ell}$.

Lemma 2.13. Assume $d \leqslant(n+2) / 2$. Then for all $\ell=0, \ldots, d$, there exist natural isomorphisms $P_{\ell} \simeq j^{*} \mathcal{O}_{\Delta}(\ell)$.

Proof. Consider the natural exact triangle $\mathcal{O}_{\Delta}(\ell)^{\prime} \rightarrow \mathcal{O}_{\Delta}(\ell) \rightarrow j^{*} \mathcal{O}_{\Delta}(\ell)$. As in the proof of [Kuz09, Proposition 3.8] (cf. Example 2.9(i)), we shall show that $\mathcal{O}_{\Delta}(\ell)^{\prime}$, for $\ell=0, \ldots, d-$ 1 , is contained in the subcategory spanned by $\left\langle\mathcal{O}_{X}(-(n+1-d)), \ldots, \mathcal{O}_{X}(-(n+1-d)+\right.$ $\ell-1)\rangle \otimes \mathrm{D}^{\mathrm{b}}(X)$ and $\mathrm{D}^{\mathrm{b}}(X) \otimes\left\langle\mathcal{O}_{X}, \ldots, \mathcal{O}_{X}(n+1-d)\right\rangle$. For this, consider the projection $k^{*}: \mathrm{D}^{\mathrm{b}}(X \times X) \rightarrow\left\langle\mathcal{O}_{X}(-(n+1-d)), \ldots, \mathcal{O}_{X}(-(n+1-d)+\ell-1)\right\rangle^{\perp} \otimes \mathcal{A}_{X}$. It suffices to show that the kernel of $\mathcal{O}_{\Delta}(\ell) \rightarrow k^{*} \mathcal{O}_{\Delta}(\ell)$ is $\mathcal{O}_{\Delta}(\ell)^{\prime}$ or, equivalently, that $k^{*} \mathcal{O}_{\Delta}(\ell) \simeq j^{*} \mathcal{O}_{\Delta}(\ell)$. Now, $\operatorname{Hom}^{*}\left(p_{1}^{*} \mathcal{O}_{X}(a), k^{*} \mathcal{O}_{\Delta}(\ell)\right)=0$ holds for $a=-(n+1-d), \ldots,-(n+1-d)+\ell-1$ by the definition of $k^{*}$ and for $a=-(n+1-d)+\ell, \ldots, 0$, as $E \longmapsto i^{*}\left(E \otimes \mathcal{O}_{X}(\ell)\right)$ is trivial on $\left\langle\mathcal{O}_{X}, \ldots, \mathcal{O}_{X}(n+1-d-\ell)\right\rangle$.

Next, convolute the above exact triangle with $\mathcal{O}_{\Delta}(1)$ from the left to obtain the exact triangle $\mathcal{O}_{\Delta}(1) \circ \mathcal{O}_{\Delta}(\ell)^{\prime} \longrightarrow \mathcal{O}_{\Delta}(\ell+1) \longrightarrow \mathcal{O}_{\Delta}(1) \circ j^{*} \mathcal{O}_{\Delta}(\ell)$. As $\mathcal{O}_{\Delta}(1) \circ \mathcal{O}_{\Delta}(\ell)^{\prime}$ is now contained in the span of $\left\langle\mathcal{O}_{X}(-(n+1-d)+1), \ldots, \mathcal{O}_{X}(-(n+1-d)+\ell)\right\rangle \otimes \mathrm{D}^{\mathrm{b}}(X)$ and $\mathrm{D}^{\mathrm{b}}(X) \otimes\left\langle\mathcal{O}_{X}, \ldots, \mathcal{O}_{X}(n+1-d)\right\rangle$, 


\section{Huybrechts and J. V. Rennemo}

it becomes trivial under $j^{*}$. This then implies that $j^{*} \mathcal{O}_{\Delta}(\ell+1) \simeq j^{*}\left(\mathcal{O}_{\Delta}(1) \circ j^{*} \mathcal{O}_{\Delta}(\ell)\right) \simeq P_{1} \circ P_{\ell} \simeq$ $P_{\ell+1}$ by induction. Note that the argument works as long as $-(n+1-d)+\ell \leqslant 0$, which under the assumption on $d$ holds for $\ell=0, \ldots, d-1$.

2.4. Consider a smooth hypersurface $X \subset \mathbb{P}:=\mathbb{P}^{n+1}$ of degree $d$. We write $\Delta_{\mathbb{P}} \subset \mathbb{P} \times \mathbb{P}$ and, as before, $\Delta=\Delta_{X} \subset X \times X$ for the two diagonals as well as for the corresponding closed immersions.

LEMMA 2.14. The pull-back of the structure sheaf $\mathcal{O}_{\Delta_{\mathbb{P}}} \in \mathrm{D}^{\mathrm{b}}(\mathbb{P} \times \mathbb{P})$ under the natural embedding $\varphi: X \times X \hookrightarrow \mathbb{P} \times \mathbb{P}$ is a complex $\varphi^{*} \mathcal{O}_{\Delta_{\mathbb{P}}} \in \mathrm{D}^{\mathrm{b}}(X \times X)$ concentrated in degrees 0 and -1 with cohomology sheaves $\mathcal{H}^{0} \simeq \mathcal{O}_{\Delta_{X}}$ and $\mathcal{H}^{-1} \simeq \mathcal{O}_{\Delta_{X}}(-d)$.

Proof. We view the cohomology sheaves $\mathcal{H}^{i}$ of $\varphi^{*} \mathcal{O}_{\Delta_{\mathbb{P}}}$ as $\mathcal{H}^{i} \simeq \mathcal{H}^{i}\left(\mathcal{O}_{X \times X} \otimes_{\mathbb{P} \times \mathbb{P}} \mathcal{O}_{\Delta_{\mathbb{P}}}\right)$. They are supported on $\Delta_{X}$ and can be computed by means of the locally free resolution

$$
E:=\left[\mathcal{O}_{\mathbb{P} \times \mathbb{P}}(-d,-d) \rightarrow \mathcal{O}_{\mathbb{P} \times \mathbb{P}}(-d, 0) \oplus \mathcal{O}_{\mathbb{P} \times \mathbb{P}}(0,-d) \rightarrow \mathcal{O}_{\mathbb{P} \times \mathbb{P}}\right] \stackrel{\sim}{\rightarrow} \mathcal{O}_{X \times X} \in \mathrm{D}^{\mathrm{b}}(\mathbb{P} \times \mathbb{P}) .
$$

Hence, $\mathcal{H}^{i} \simeq \mathcal{H}^{i}\left(E \otimes_{\mathbb{P} \times \mathbb{P}} \mathcal{O}_{\Delta_{\mathbb{P}}}\right) \simeq \mathcal{H}^{i}\left[\mathcal{O}_{\Delta_{\mathbb{P}}}(-2 d) \rightarrow \mathcal{O}_{\Delta_{\mathbb{P}}}(-d)^{\oplus 2} \rightarrow \mathcal{O}_{\Delta_{\mathbb{P}}}\right]$ and, in particular, $\mathcal{H}^{i}=0$ for $i \neq 0,-1,-2$. Moreover, as $\mathcal{H}^{-2}$ is supported on the proper subscheme $\Delta \subset \Delta_{\mathbb{P}}$ and is, at the same time, contained in $\mathcal{O}_{\Delta_{\mathbb{P}}}(-2 d)$, it has to be trivial, too. Obviously, $\mathcal{H}^{0} \simeq \mathcal{O}_{\Delta_{X}}$ and, therefore,

$$
\mathcal{H}^{-1} \simeq \operatorname{Coker}\left(\mathcal{O}_{\Delta_{\mathbb{P}}}(-2 d) \hookrightarrow \mathcal{O}_{\Delta_{\mathbb{P}}}(-d)\right) \simeq \mathcal{O}_{\Delta_{X}}(-d) .
$$

The usual exact triangle $\mathcal{H}^{-1}[1] \longrightarrow \varphi^{*} \mathcal{O}_{\Delta_{\mathbb{P}}} \rightarrow \mathcal{H}^{0}$ for a complex concentrated in degrees 0 and -1 twisted by $\mathcal{O}(d, 0)$ (or, equivalently, $\mathcal{O}(0, d)$ ) becomes

$$
\varphi^{*} \mathcal{O}_{\Delta_{\mathbb{P}}}(d) \longrightarrow \mathcal{O}_{\Delta_{X}}(d) \stackrel{\alpha}{\longrightarrow} \mathcal{O}_{\Delta_{X}}[2] .
$$

The proof of the following result is close in spirit to Kuznetsov's arguments in the proof of [Kuz04, Lemma 4.2].

Lemma 2.15. Assume $d \leqslant(n+2) / 2$. Then under the left adjoint $j^{*}: \mathrm{D}^{\mathrm{b}}(X \times X) \rightarrow \mathcal{A}_{X}(-(n+$ $1-d)) \otimes \mathcal{A}_{X}$ of the natural inclusion (see (2.3)), the object $\varphi^{*} \mathcal{O}_{\Delta_{\mathbb{P}}}(d)$ becomes trivial; that is,

$$
j^{*} \varphi^{*} \mathcal{O}_{\Delta_{\mathbb{P}}}(d) \simeq 0 .
$$

Proof. The Koszul resolution

$$
\left[\mathcal{O}_{\mathbb{P}}(-(n+1)) \otimes \Omega_{\mathbb{P}}^{n+1}(n+1) \rightarrow \cdots \rightarrow \mathcal{O}_{\mathbb{P}}(-1) \otimes \Omega_{\mathbb{P}}(1) \rightarrow \mathcal{O}_{\mathbb{P} \times \mathbb{P}}\right] \stackrel{\sim}{\rightarrow} \mathcal{O}_{\Delta_{\mathbb{P}}}
$$

allows one to compute $\varphi^{*} \mathcal{O}_{\Delta_{\mathbb{P}}}(d)$ as $\left[E_{n+1} \longrightarrow \cdots \rightarrow E_{0}\right]$, where $E_{i}:=\left.\mathcal{O}_{X}(d-i) \otimes \Omega_{\mathbb{P}}^{i}(i)\right|_{X}$.

Now, from the Euler sequence $0 \longrightarrow \Omega_{\mathbb{P}}(1) \longrightarrow \mathcal{O}_{\mathbb{P}}^{\oplus n+2} \longrightarrow \mathcal{O}_{\mathbb{P}}(1) \longrightarrow 0$ and its alternating powers $0 \longrightarrow \Omega_{\mathbb{P}}^{i}(i) \longrightarrow \mathcal{O}_{\mathbb{P}}^{\oplus\left(\begin{array}{c}n+2 \\ i\end{array}\right)} \rightarrow \Omega_{\mathbb{P}}^{i-1}(i) \longrightarrow 0$, one deduces $E_{i} \in \mathrm{D}^{\mathrm{b}}(X) \otimes\left\langle\mathcal{O}_{X}, \ldots, \mathcal{O}_{X}(n+1-d)\right\rangle$ for $i=0, \ldots, n+1-d$. As $d \leqslant(n+2) / 2$, the remaining $E_{i}$, for $i=n+2-d, \ldots, n+1$, are all contained in $\left\langle\mathcal{O}_{X}(-(n+1-d)), \ldots, \mathcal{O}_{X}\right\rangle \otimes \mathrm{D}^{\mathrm{b}}(X)$. Altogether, this shows that $\varphi^{*} \mathcal{O}_{\Delta_{\mathbb{P}}}(d)$ is contained in the left orthogonal of $\mathcal{A}_{X}(-(n+1-d)) \otimes \mathcal{A}_{X}$ and hence $j^{*} \varphi^{*} \mathcal{O}_{\Delta_{\mathbb{P}}}(d) \simeq 0$.

Combining this with Lemma 2.13 yields the next result, which again is just the kernel version of a result of Kuznetsov [Kuz04, Kuz18].

Corollary 2.16. For $d \leqslant(n+2) / 2$, one has

$$
P_{d} \simeq j^{*} \mathcal{O}_{\Delta_{X}}(d) \simeq j^{*} \mathcal{O}_{\Delta_{X}}[2] \text { and }(d) \simeq[2]
$$




\section{HOCHSCHILD COHOMOLOGY VERSUS THE JACOBIAN RING}

and, more generally,

$$
P_{d \ell+a} \simeq j^{*} \mathcal{O}_{\Delta}(a)[2 \ell] \text { and }(d \ell+a) \simeq(a) \circ[2 \ell]
$$

for all $\ell \geqslant 0$ and $0 \leqslant a<d$.

Remark 2.17. Note that this, a posteriori, shows that (1): $\mathcal{A}_{X} \rightarrow \mathcal{A}_{X}$ is an auto-equivalence.

Remark 2.18. For the reader's convenience, we briefly mention that at least for cubic fourfolds $X \subset \mathbb{P}^{5}$, the arguments presented so far already ensure that $\mathcal{A}_{X}$ is a two-dimensional Calabi-Yau category. Indeed, in this case, Lemma 2.13 applies to $\ell=n+2-d$ and hence for all $E, F \in \mathcal{A}_{X}$ one finds $\operatorname{Hom}_{\mathcal{A}_{X}}\left(i^{*}\left(E \otimes \mathcal{O}_{X}(n+2-d)\right), F\right) \simeq \operatorname{Hom}_{\mathrm{D}^{\mathrm{b}}(X)}\left(E \otimes \mathcal{O}_{X}(n+2-d), F\right) \simeq \operatorname{Hom}_{\mathrm{D}^{\mathrm{b}}(X)}(F, E[n])^{*}$, which can be read as $S_{\mathcal{A}_{X}} \circ(n+2-d) \simeq[n]$. This together with the isomorphism $(d) \simeq[2]$ yields the assertion.

2.5. We shall need an alternative description of the isomorphism $j^{*} \mathcal{O}_{\Delta}(d) \simeq j^{*} \mathcal{O}_{\Delta}[2]$ that involves the tangent bundle $\mathcal{T}_{X}$.

The normal bundle sequence $\left.0 \longrightarrow \mathcal{T}_{X} \rightarrow \mathcal{T}_{\mathbb{P}}\right|_{X} \rightarrow \mathcal{O}_{X}(d) \rightarrow 0$ is encoded by the boundary map $\mathcal{O}_{X}(d) \rightarrow \mathcal{T}_{X}$ [1], which is certainly non-trivial for $d>2$ and $n>2$, which covers all cases of interest to us. Taking the direct images under the diagonal morphism yields

$$
\mathcal{O}_{\Delta}(d) \rightarrow \Delta_{*} \mathcal{T}_{X}[1]
$$

The boundary morphism of the short exact sequence $0 \rightarrow \Delta_{*} \Omega_{X} \rightarrow \mathcal{O}_{X \times X} / \mathcal{I}_{\Delta}^{2} \rightarrow \mathcal{O}_{\Delta} \longrightarrow 0$ is the universal Atiyah class At: $\mathcal{O}_{\Delta} \rightarrow \Delta_{*} \Omega_{X}[1]$. Taking exterior powers, it yields a natural map $\bigoplus \mathrm{At}^{p}: \mathcal{O}_{\Delta} \rightarrow \Delta_{*} \bigoplus_{p=0}^{n} \Omega_{X}^{p}[p]$, whose adjoint

$$
\Delta^{*} \mathcal{O}_{\Delta} \stackrel{\sim}{\longrightarrow} \bigoplus_{p=0}^{n} \Omega_{X}^{p}[p]
$$

is known to be an isomorphism [Că105, Mar09]. We shall rather work in the dual setting [Kuz09, Section 8]:

$$
\bigoplus_{p=0}^{n} \bigwedge^{p} \mathcal{T}_{X}[-p] \stackrel{\sim}{\longrightarrow} \Delta ! \mathcal{O}_{\Delta}
$$

Taking the direct image under the diagonal and composing with the natural inclusion of $\mathcal{T}_{X}[-1]$ on the left and with the adjunction $\Delta_{*} \Delta^{!} \longrightarrow$ id on the right yields

$$
\Delta_{*} \mathcal{T}_{X}[1] \rightarrow \Delta_{*} \bigoplus_{p=0}^{n} \bigwedge^{p} \mathcal{T}_{X}[2-p] \stackrel{\sim}{\rightarrow} \Delta_{*} \Delta ! \mathcal{O}_{\Delta}[2] \rightarrow \mathcal{O}_{\Delta}[2]
$$

Now, composing (2.8) and (2.9) yields a map

$$
\beta: \mathcal{O}_{\Delta}(d) \rightarrow \Delta_{*} \mathcal{T}_{X}[1] \rightarrow \mathcal{O}_{\Delta}[2],
$$

which can be compared to $\alpha$ in (2.7).

Lemma 2.19. The two maps $\alpha, \beta: \mathcal{O}_{\Delta}(d) \rightarrow \Delta_{*} \mathcal{T}_{X}[1] \rightarrow \mathcal{O}_{\Delta}[2]$ coincide (up to non-trivial scaling).

Proof. This can be seen as a consequence of [HT10, Theorem 2.10]. Indeed, $\beta$ is by construction the composition of the universal Atiyah class with the Kodaira-Spencer class for the embedding $X \subset \mathbb{P}^{n+1}$, which coincides with the universal obstruction class $\alpha$. 


\section{Huybrechts And J. V. Rennemo}

Alternatively, one can show that $\operatorname{Hom}_{X \times X}\left(\mathcal{O}_{\Delta}(d), \mathcal{O}_{\Delta}[2]\right)$ is just one-dimensional and that both maps $\alpha$ and $\beta$ are non-zero. Indeed, $\operatorname{Hom}_{X \times X}\left(\mathcal{O}_{\Delta}(d), \mathcal{O}_{\Delta}[2]\right) \simeq H^{0}\left(X, \Delta !_{\mathcal{O}_{\Delta}}(-d)[2]\right) \simeq$ $H^{0}\left(X, \bigoplus_{p=0}^{n} \bigwedge^{p} \mathcal{T}_{X}(-d)[2-p]\right)$. For degree reasons, only $H^{2}(X, \mathcal{O}(-d)), H^{1}\left(X, \mathcal{T}_{X}(-d)\right)$, and $H^{0}\left(X, \bigwedge^{2} \mathcal{T}_{X}(-d)\right)$ contribute to the direct sum. The first and third cohomology groups are obviously trivial, and due to the normal bundle sequence, the second one is one-dimensional. Clearly, $\alpha \neq 0$, as $j^{*} \alpha$ is an isomorphism by Lemma 2.15. Similarly, one checks that $\beta \neq 0$, as otherwise the adjunction $\Delta ! \Delta_{*} \mathcal{O}_{\Delta} \longrightarrow \mathcal{O}_{\Delta}$ would be zero. Hence, $\alpha$ and $\beta$ differ at most by a non-trivial scalar.

Remark 2.20. The interpretation of $\alpha$ as the universal obstruction class has the following geometric consequence for objects in $\mathcal{A}_{X}$ : non-trivial objects $E \in \mathcal{A}_{X}$ are maximally obstructed; that is, they do not even deform to first order to the ambient projective space. Indeed, $\alpha$ as a morphism between Fourier-Mukai kernels applied to any object $E \in \mathrm{D}^{\mathrm{b}}(X)$ yields the obstruction $o(E) \in \operatorname{Ext}^{2}(E \otimes \mathcal{O}(d), E)$ to extending $E$ to the first-order neighbourhood of $X$ in $\mathbb{P}$; cf. [HT10]. However, for $E \in \mathcal{A}_{X}$, this class, via adjunction, yields the isomorphism $E(d) \simeq i^{*}(E \otimes \mathcal{O}(d)) \stackrel{\sim}{\longrightarrow} E[2]$, and so $o(E) \neq 0$ for all non-trivial $E \in \mathcal{A}_{X}$.

Composing $\left.\mathcal{O}_{X}^{\oplus n+2} \longrightarrow \mathcal{T}_{\mathbb{P}}(-1)\right|_{X}$, coming from the restriction of the Euler sequence, with the natural projection $\left.\mathcal{T}_{\mathbb{P}}(-1)\right|_{X} \longrightarrow \mathcal{O}_{X}(d-1)$ in the normal bundle sequence yields a map

$$
\gamma:\left.\mathcal{O}_{X}^{\oplus n+2} \longrightarrow \mathcal{T}_{\mathbb{P}}(-1)\right|_{X} \longrightarrow \mathcal{O}_{X}(d-1) .
$$

This map is induced by the partial derivatives $\partial_{i} f \in H^{0}\left(X, \mathcal{O}_{X}(d-1)\right)$ of the equation $f \in$ $k\left[x_{0}, \ldots, x_{n+1}\right]_{d}$ defining $X \subset \mathbb{P}^{n+1}$.

Corollary 2.21. The induced map $j^{*} \Delta_{*} \gamma$ in the product category $\mathcal{A}_{X}(-(n+1-d)) \otimes \mathcal{A}_{X} \subset$ $\mathrm{D}^{\mathrm{b}}(X \times X)$ is trivial:

$$
0=j^{*} \Delta_{*} \gamma: j^{*} \mathcal{O}_{\Delta}^{\oplus n+2} \longrightarrow j^{*} \mathcal{O}_{\Delta}(d-1) .
$$

Proof. First note that the composition $\alpha(-1) \circ \Delta_{*} \gamma: \mathcal{O}_{\Delta}^{\oplus n+2} \rightarrow \mathcal{O}_{\Delta}(d-1) \rightarrow \mathcal{O}_{\Delta}(-1)[2]$ is trivial. Indeed, as $\alpha=\beta$ and $\beta$ factors through the boundary map $\mathcal{O}_{X}(d-1) \rightarrow \mathcal{T}_{X}(-1)[1]$ of the normal bundle sequence, this follows from the observation that already the composition $\left.\mathcal{T}_{\mathbb{P}}(-1)\right|_{X} \rightarrow \mathcal{O}_{X}(d-1) \rightarrow \mathcal{T}_{X}(-1)[1]$ is trivial. Hence, $\Delta_{*} \gamma$ factors through a morphism $\delta: \mathcal{O}_{\Delta}^{\oplus n+2} \longrightarrow \varphi^{*} \mathcal{O}_{\Delta_{\mathbb{P}}}(d-1)$ (use the triangle $(2.7)$ tensored by $\mathcal{O}_{X}(-1)$ ), and it suffices to show that $j^{*} \delta=0$.

Now, using the notation from the proof of Lemma 2.15, we let $E_{i}^{\prime}:=E_{i} \otimes \mathcal{O}(-1,0)$. Then we still have $E_{i}^{\prime} \in \mathrm{D}^{\mathrm{b}}(X) \otimes\left\langle\mathcal{O}_{X}, \ldots, \mathcal{O}_{X}(n+1-d)\right\rangle$ for $i=0, \ldots, n+1-d$ and $E_{i}^{\prime} \in\left\langle\mathcal{O}_{X}(-(n+1-\right.$ $\left.d)), \ldots, \mathcal{O}_{X}\right\rangle \otimes \mathrm{D}^{\mathrm{b}}(X)$ for $i=n+2-d, \ldots, n$. Only the last one, $E_{n+1}^{\prime} \simeq \mathcal{O}(d-1-(n+1),-1)$, does not vanish under $j^{*}$. Hence, the pull-back under $j^{*}$ of the natural map $\varphi^{*} \mathcal{O}_{\Delta_{\mathbb{P}}}(d-1) \longrightarrow E_{n+1}^{\prime}[n+1]$ becomes an isomorphism $j^{*} \varphi^{*} \mathcal{O}_{\Delta_{\mathbb{P}}}(d-1) \stackrel{\sim}{\longrightarrow} j^{*} E_{n+1}^{\prime}[n+1]$. Therefore, in order to prove the assertion, it suffices to show that the composition $\mathcal{O}_{\Delta}^{\oplus n+2} \rightarrow \varphi^{*} \mathcal{O}_{\Delta_{\mathbb{P}}}(d-1) \rightarrow E_{n+1}^{\prime}[n+1]$ is trivial, which follows from $\operatorname{Ext}_{X \times X}^{n+1}\left(\mathcal{O}_{\Delta}, E_{n+1}^{\prime}\right) \simeq H^{n-1}\left(X, \mathcal{O}_{X}(d-n-1)\right)^{*}=0$ using Serre duality.

See Section 6 for an interpretation of his result using the category of graded matrix factorizations. 


\section{HOCHSCHILD COHOMOLOGY VERSUS THE JACOBIAN RING}

\section{Extended Hochschild cohomology of hypersurfaces}

We define the Hochschild cohomology $\mathrm{HH}^{*}\left(\mathcal{A}_{X},(1)\right)$ of the category $\mathcal{A}_{X}$ endowed with the degreeshift functor (1) associated with any smooth hypersurface $X \subset \mathbb{P}^{n+1}$. It is intimately related to the usual even Hochschild cohomology $\operatorname{HH}^{*}\left(\mathcal{A}_{X}\right)$ but also incorporates the degree-shift functor. This section also contains the comparison of the Jacobian ring $J(X)$ with $\mathrm{HH}^{*}\left(\mathcal{A}_{X},(1)\right.$ ) (cf. Theorem 1.1). We work again over an arbitrary field of characteristic zero.

3.1. As before, we let $X \subset \mathbb{P}=\mathbb{P}^{n+1}$ be a smooth hypersurface defined by a homogeneous polynomial $f \in k\left[x_{0}, \ldots, x_{n+1}\right]_{d}$ of degree $d>2$. We set $\sigma:=(n+2)(d-2)$ and define

$$
L(X):=\bigoplus_{\ell=0}^{\sigma} L_{\ell}(X)=\bigoplus_{\ell=0}^{\sigma} \operatorname{Hom}\left(P_{0}, P_{\ell}\right)
$$

(with Hom taken in the full subcategory $\mathcal{A}_{X}(-(n+1-d)) \otimes \mathcal{A}_{X} \subset \mathrm{D}^{\mathrm{b}}(X \times X)$ ) with its natural ring structure defined by composition. More precisely, by applying convolution with $P_{\ell}$ as defined in (2.6), one obtains a natural map $\operatorname{Hom}\left(P_{0}, P_{\ell^{\prime}}\right) \rightarrow \operatorname{Hom}\left(P_{\ell} \circ P_{0}, P_{\ell} \circ P_{\ell^{\prime}}\right) \simeq \operatorname{Hom}\left(P_{\ell}, P_{\ell+\ell^{\prime}}\right)$, which then yields

$$
\operatorname{Hom}\left(P_{0}, P_{\ell}\right) \times \operatorname{Hom}\left(P_{0}, P_{\ell^{\prime}}\right) \rightarrow \operatorname{Hom}\left(P_{0}, P_{\ell}\right) \times \operatorname{Hom}\left(P_{\ell}, P_{\ell+\ell^{\prime}}\right) \rightarrow \operatorname{Hom}\left(P_{0}, P_{\ell+\ell^{\prime}}\right)
$$

with the convention that the multiplication is trivial as soon as $\ell+\ell^{\prime}$ exceeds $\sigma$. Standard arguments show that this endows $L(X)$ with the structure of a graded ring.

Next, consider the natural map

$$
H^{0}\left(\mathbb{P}, \mathcal{O}_{\mathbb{P}}(1)\right) \simeq H^{0}\left(X, \mathcal{O}_{X}(1)\right) \stackrel{\sim}{\rightarrow} \operatorname{Hom}\left(\mathcal{O}_{\Delta}, \mathcal{O}_{\Delta}(1)\right) \rightarrow \operatorname{Hom}\left(j^{*} \mathcal{O}_{\Delta}, j^{*} \mathcal{O}_{\Delta}(1)\right)=L_{1}(X) .
$$

Lemma 3.1. The homomorphism (3.1) is bijective.

Proof. (1) We shall first show that (3.1) is bijective for $d \geqslant 4$ and injective for $d \geqslant 3$. Consider the exact triangle $P_{1}^{\prime} \longrightarrow \mathcal{O}_{\Delta}(1) \longrightarrow P_{1}$. Using (2.5), a direct computation shows that

$$
P_{1} \simeq\left[H^{0}\left(X, \mathcal{O}_{X}(1)\right)^{\otimes m+1} \otimes \mathcal{O}(-m, 0) \rightarrow \cdots \rightarrow \bigoplus_{i=-1}^{m} \mathcal{O}(-i, i+1) \rightarrow \mathcal{O}_{\Delta}(1)\right]
$$

where one uses that all pull-backs, direct images, and tensor products are in fact underived due to the vanishing $H^{>0}(X, \mathcal{O}(i))=0$. Hence,

$$
\operatorname{Hom}\left(\mathcal{O}_{\Delta}, P_{1}^{\prime}[i]\right) \simeq \operatorname{Hom}\left(\mathcal{O}_{X}, \Delta^{*} P_{1}^{\prime} \otimes \omega_{X}^{*}[i-n]\right)=0
$$

for $i-n<-m-1$ or, equivalently, for $i<d-2$. Therefore, $\operatorname{Hom}\left(\mathcal{O}_{\Delta}, P_{1}^{\prime}\right)=0=\operatorname{Hom}\left(\mathcal{O}_{\Delta}, P_{1}^{\prime}[1]\right)$ for $d \geqslant 4$, which proves the bijectivity of (3.1) in these cases. For $d=3$, we still have $\operatorname{Hom}\left(\mathcal{O}_{\Delta}, P_{1}^{\prime}\right)$ $=0$, which proves at least the injectivity. ${ }^{4}$

(2) Surjectivity also holds for $d=3$, but since the calculation is long and we do not have any applications for $n>4$, we only give the proof in the case $n=4$. For this, we will prove that in (3.1) one has $\operatorname{dim} H^{0}\left(X, \mathcal{O}_{X}(1)\right) \geqslant \operatorname{dim} L_{1}(X)$, which, together with the injectivity from part (1), proves the bijectivity.

By definition and Serre duality for the Calabi-Yau category $\mathcal{A}_{X}(-2) \otimes \mathcal{A}_{X}$ (which is of dimension four $), L_{1}(X)=\operatorname{Hom}\left(j^{*} \mathcal{O}_{\Delta}, j^{*} \mathcal{O}_{\Delta}(1)\right) \simeq \operatorname{Ext}^{4}\left(j^{*} \mathcal{O}_{\Delta}(1), j^{*} \mathcal{O}_{\Delta}\right)^{*} \simeq \operatorname{Ext}^{4}\left(\mathcal{O}_{\Delta}(1), j^{*} \mathcal{O}_{\Delta}\right)^{*} \simeq$

\footnotetext{
${ }^{4}$ Note that for $d \mid(n+2)$ and $d<n+2$, the injectivity also follows from Proposition 3.5.
} 


\section{Huybrechts and J. V. Rennemo}

$H^{4}\left(X, \Delta^{!} j^{*} \mathcal{O}_{\Delta} \otimes \mathcal{O}_{X}(-1)\right)^{*} \simeq H^{0}\left(X, \Delta^{*} j^{*} \mathcal{O}_{\Delta} \otimes \mathcal{O}_{X}(2)\right)^{*}$, where we use that $\Delta^{!} \simeq\left(\omega_{X}^{*} \otimes()\right) \circ$ $\Delta^{*} \circ[-4]$.

In order to compute $\Delta^{*} j^{*} \mathcal{O}_{\Delta}$, consider the left projections $j_{0}^{*}: \mathrm{D}^{\mathrm{b}}(X \times X) \rightarrow \mathrm{D}^{\mathrm{b}}(X) \otimes$ $\left\langle\mathcal{O}_{X}(2)\right\rangle^{\perp}$ and $j_{1}^{*}: \mathrm{D}^{\mathrm{b}}(X \times X) \rightarrow \mathrm{D}^{\mathrm{b}}(X) \otimes\left\langle\mathcal{O}_{X}(1), \mathcal{O}_{X}(2)\right\rangle^{\perp}$. Then $j^{*} \mathcal{O}_{\Delta}$ is recursively described by the exact triangles

$$
\begin{aligned}
\mathcal{O}_{\Delta} \circ \mathcal{O}(-2,2) & \longrightarrow \mathcal{O}_{\Delta} \longrightarrow j_{0}^{*} \mathcal{O}_{\Delta}, \\
j_{0}^{*} \mathcal{O}_{\Delta} \circ \mathcal{O}(-1,1) & \longrightarrow j_{0}^{*} \mathcal{O}_{\Delta} \longrightarrow j_{1}^{*} \mathcal{O}_{\Delta}, \\
j_{1}^{*} \mathcal{O}_{\Delta} \circ \mathcal{O} & \longrightarrow j_{1}^{*} \mathcal{O}_{\Delta} \longrightarrow j^{*} \mathcal{O}_{\Delta} .
\end{aligned}
$$

Applying $\Delta^{*}$ yields the diagram of exact triangles

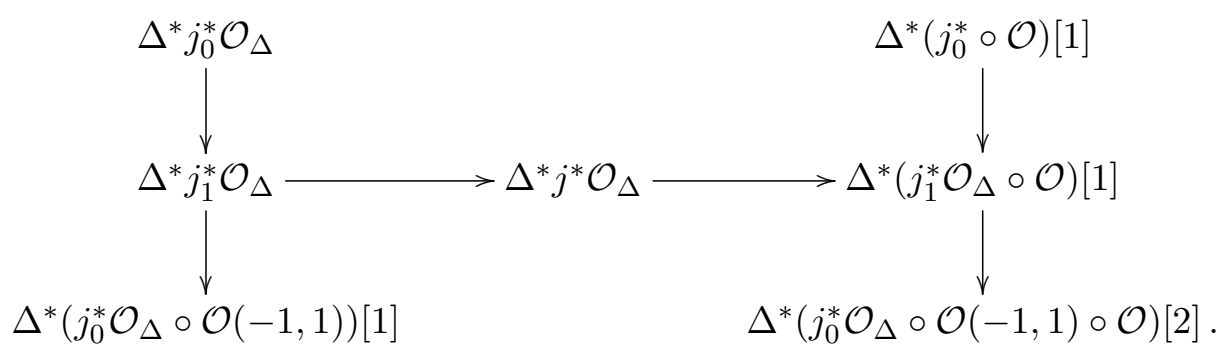

The computation of the four corners is straightforward. For example, the lower-left corner is $\left.\Delta^{*}\left(j_{0}^{*} \mathcal{O}_{\Delta} \otimes \mathcal{O}(-1,1)\right) \simeq \Omega_{\mathbb{P}}\right|_{X}[1]$, as it is the cone of the natural map

$$
H^{0}\left(X, \mathcal{O}_{X}(1)\right) \otimes \mathcal{O}_{X}(-1) \simeq \Delta^{*}\left(\mathcal{O}_{\Delta} \circ \mathcal{O}(-2,2) \circ \mathcal{O}(-1,1)\right) \rightarrow \Delta^{*}\left(\mathcal{O}_{\Delta} \circ \mathcal{O}(-1,1)\right) \simeq \mathcal{O}_{X} .
$$

Similarly, the lower-right corner is $\Delta^{*}\left(j_{0}^{*} \mathcal{O}_{\Delta} \circ \mathcal{O}(-1,1) \circ \mathcal{O}\right)[2] \simeq\left(\left.\Omega_{\mathbb{P}}\right|_{X}(-1) \otimes H^{0}\left(X, \mathcal{O}_{X}(1)\right)\right)[3]$. Indeed, it is the [2]-shift of the cone of the map

$$
H^{0}\left(X, \mathcal{O}_{X}(1)\right)^{\otimes 2} \otimes \mathcal{O}_{X}(-2) \simeq \Delta^{*}\left(\mathcal{O}_{\Delta} \circ \mathcal{O}(-2,2) \circ \mathcal{O}(-1,1) \circ \mathcal{O}\right) \longrightarrow \Delta^{*}\left(\mathcal{O}_{\Delta} \circ \mathcal{O}(-1,1) \circ \mathcal{O}\right),
$$

which is the evaluation $H^{0}\left(X, \mathcal{O}_{X}(1)\right) \otimes \mathcal{O}_{X}(-2) \rightarrow \mathcal{O}_{X}(-1)$ tensored with $H^{0}\left(X, \mathcal{O}_{X}(1)\right)$. For the computation of the upper-left corner, use $\Delta^{*} \mathcal{O}_{\Delta} \simeq \bigoplus \Omega_{X}^{p}[p]$ and $\Delta^{*}\left(\mathcal{O}_{\Delta} \circ \mathcal{O}(-2,2)\right) \simeq \mathcal{O}_{X}$ to conclude that $\Delta^{*}\left(j_{0}^{*} \mathcal{O}_{\Delta}\right) \simeq \bigoplus_{p \geqslant 1} \Omega_{X}^{p}[p]$. Finally, the upper-right corner is the [1]-shift of the cone of

$$
H^{0}\left(X, \mathcal{O}_{X}(2)\right) \otimes \mathcal{O}_{X}(-2) \simeq \Delta^{*}\left(\mathcal{O}_{\Delta} \circ \mathcal{O}(-2,2) \circ \mathcal{O}\right) \rightarrow \Delta^{*}\left(\mathcal{O}_{\Delta} \circ \mathcal{O}\right) \simeq \mathcal{O}_{X}
$$

and so $\left.\Delta^{*}\left(j_{0}^{*} \circ \mathcal{O}\right)[1] \simeq \Omega_{\mathbb{P}^{\prime}}\right|_{X}[2]$, where $X \subset \mathbb{P}^{\prime}=\mathbb{P}^{20}$ is the second Veronese embedding.

Now, in order to compute $H^{0}\left(X, \Delta^{*} j^{*} \mathcal{O}_{\Delta} \otimes \mathcal{O}_{X}(2)\right)$, use the induced diagram of triangles

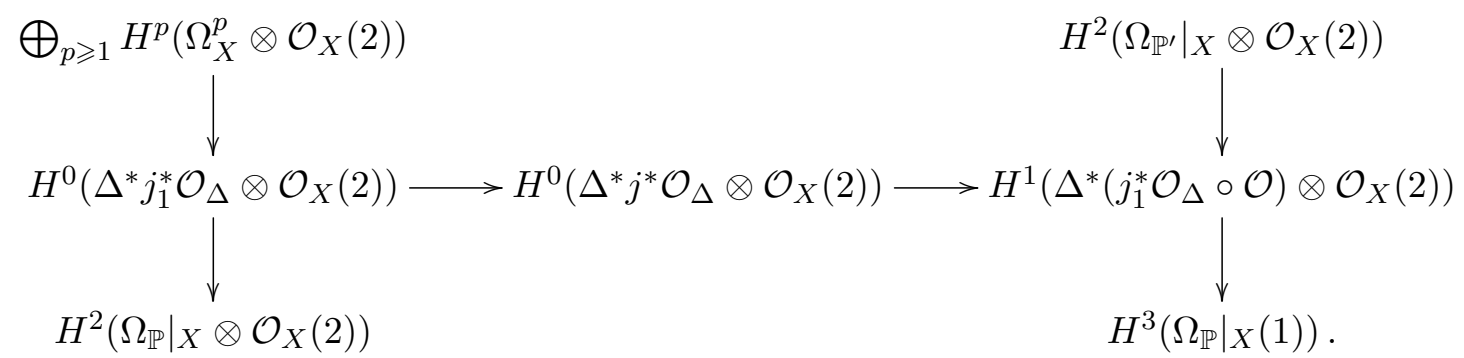

Using the Euler and conormal bundle sequences (and their exterior powers) and standard vanishing results, one easily shows that the only non-trivial term in the four corners is the term $H^{2}\left(X, \Omega_{X}^{2} \otimes \mathcal{O}_{X}(2)\right) \simeq H^{3}\left(X, \Omega_{X}(-1)\right) \simeq H^{4}\left(X, \mathcal{O}_{X}(-4)\right) \simeq H^{0}\left(X, \mathcal{O}_{X}(1)\right)^{*}$ in the left-upper corner. 


\section{HOCHSCHILD COHOMOLOGY VERSUS THE JACOBIAN RING}

Remark 3.2. Note that the analogous maps $H^{0}(X, \mathcal{O}(\ell)) \rightarrow L_{\ell}(X)$ for arbitrary $\ell$ are usually not surjective. For example, in the case of the cubic fourfold, $L_{3}(X)$ is of dimension 22 , but by Proposition 3.3, the map $H^{0}\left(X, \mathcal{O}_{X}(3)\right) \rightarrow L_{3}(X)$ factors through $J_{3}(X) \simeq H^{1}\left(X, \mathcal{T}_{X}\right)$ (see below) which is of dimension 20 .

The map (3.1) induces a homomorphism of graded rings

$$
R:=k\left[x_{0}, \ldots, x_{n+1}\right] \rightarrow L(X) .
$$

For this, one has to check that the images of $x_{i}$ and $x_{j}$ in $L(X)$ commute, which follows from the commutativity of the diagram

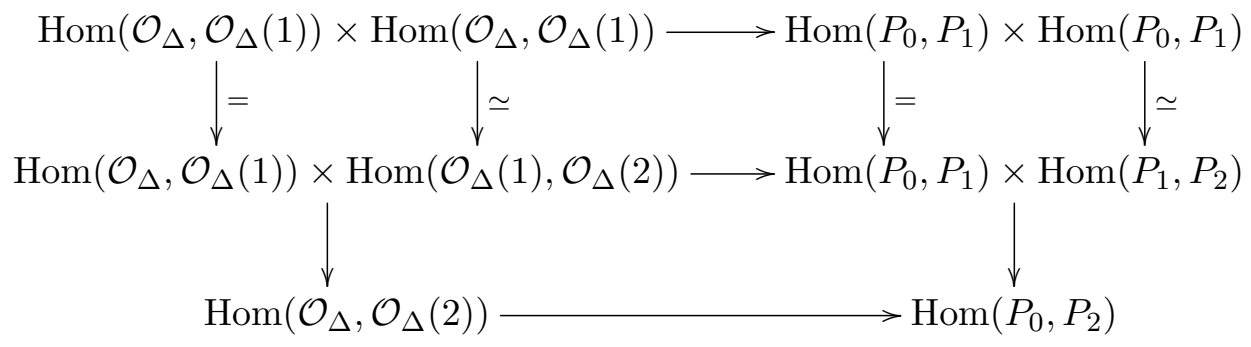

and the fact that the compositions $x_{i} \circ x_{j}$ and $x_{j} \circ x_{i}$ in $\operatorname{Hom}\left(\mathcal{O}_{\Delta}, \mathcal{O}_{\Delta}(2)\right)=H^{0}\left(X, \mathcal{O}_{X}(2)\right)$ correspond to $x_{i} x_{j}=x_{j} x_{i}$ and so coincide.

The following observation essentially proves the first half of Theorem 1.1; see also Corollary 3.7. For related results in this direction, compare with [BFK12, Proposition 5.14].

Proposition 3.3. If $d \leqslant(n+2) / 2$, the ring homomorphism (3.3) factors through a graded-ring homomorphism from the Jacobian ring $J(X)=R /\left(\partial_{i} f\right)$ to $L(X)$ :

$$
R(X) \longrightarrow J(X) \stackrel{\pi}{\longrightarrow} L(X) .
$$

Proof. This is an immediate consequence of Corollary 2.21, which claims that all partial derivatives $\partial_{i} f \in R_{d-1}$ of the equation $f$ defining $X$ vanish under (3.3). At this point, one uses Lemma 2.13 to ensure that $P_{d-1} \simeq j^{*} \mathcal{O}_{\Delta}(d-1)$, for which the assumption on $d$ is needed.

3.2. In this section, we in addition assume $d \mid(n+2)$ with $d<n+2$. Then, in particular, Kuznetsov's Theorem 2.1, Corollary 2.4, Lemma 2.13, and Corollary 2.16 all apply to our situation. It turns out that the Jacobian ring $J(X)$ and $L(X)$ (apart from possibly being noncommutative) are both Gorenstein rings of the same top degree $\sigma=(n+2)(d-2)$; cf. Remark 2.2.

Proposition 3.4. The ring $L(X)$ has the following properties:

(i) $L_{0}(X) \simeq \operatorname{HH}^{0}\left(\mathcal{A}_{X}\right) \simeq k$.

(ii) $L_{d \ell}(X)=\operatorname{Hom}\left(P_{0}, P_{d \ell}\right) \simeq \operatorname{Hom}\left(j^{*} \mathcal{O}_{\Delta}, j^{*} \mathcal{O}_{\Delta}[2 \ell]\right) \simeq \mathrm{HH}^{2 \ell}\left(\mathcal{A}_{X}\right)$, which is of dimension one for $d \ell=\sigma$.

(iii) Composition induces a non-degenerate pairing $L_{\ell}(X) \times L_{\sigma-\ell}(X) \rightarrow L_{\sigma}(X) \simeq k$.

Proof. Recall from Theorem 2.1 that $\sigma / d$ is the dimension of the Calabi-Yau category $\mathcal{A}_{X}$.

In property (i), the first isomorphism is by definition and the second one follows from [Kuz09, Corollary 7.5]. Alternatively, use the arguments in the proof of Lemma 3.1.

Next, property (ii) follows from Corollary 2.16 and the definition of Hochschild cohomology; see [Kuz09]. That in property (ii), the space is of dimension one for $d \ell=\sigma$ can be deduced either 


\section{Huybrechts And J. V. Rennemo}

from Serre duality for the $(2 \sigma / d)$-dimensional Calabi-Yau category $\mathcal{A}_{X}(-(n+1-d)) \otimes \mathcal{A}_{X}(\mathrm{cf}$. Corollary 2.4) or from [Kuz18, Proposition 5.3] and [Kuz09, Corollary 7.5] showing that

$$
\mathrm{HH}^{2 \operatorname{dim} \mathcal{A}_{X}}\left(\mathcal{A}_{X}\right) \simeq \mathrm{HH}_{-\operatorname{dim} \mathcal{A}_{X}}\left(\mathcal{A}_{X}\right) \simeq \mathrm{HH}_{-\operatorname{dim} \mathcal{A}_{X}}(X) \simeq \bigoplus H^{-\operatorname{dim} \mathcal{A}_{X}+p}\left(X, \Omega_{X}^{p}\right),
$$

which can be shown to be one-dimensional (using Hirzebruch's formula for $\chi\left(\Omega_{X}^{p}\right)$, see [SGA7], and the Lefschetz hyperplane theorem). Yet another possibility would be to follow the arguments in the proof of Lemma 3.1.

The last assertion follows again from Serre duality and the fact that $\mathcal{A}_{X}(-(n+1-d)) \otimes \mathcal{A}_{X}$ is a Calabi-Yau category of dimension $2 \operatorname{dim}\left(\mathcal{A}_{X}\right)=2 \sigma / d$; cf. Corollary 2.4.

In fact, it seems likely that $\operatorname{Hom}\left(P_{0}, P_{\ell}\right)=0$ for all $\ell>\sigma$, which certainly is the case if $d \mid \ell$. So presumably, $L(X)=\bigoplus_{\ell \geqslant 0} \operatorname{Hom}\left(P_{0}, P_{\ell}\right)$, but this is of no importance for what follows.

Proposition 3.5. The ring homomorphism $\pi: J(X) \rightarrow L(X)$ in (3.4) is injective.

Proof. For a proof of the injectivity in degree one, see the proof of Lemma 3.1. However, it can also be seen as a consequence of the following arguments.

The graded-ring homomorphism $\pi$ induces commutative diagrams

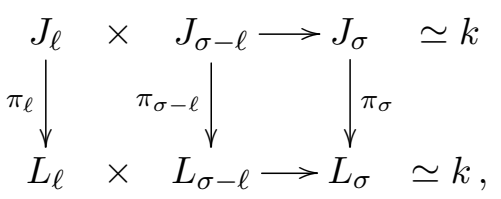

with both rows non-degenerate, due to [Don83, Theorem 2.5] and Proposition 3.4(iii). Hence, the injectivity of $\pi$ is equivalent to the injectivity of $\pi_{\sigma}$, which in turn is equivalent to $\pi_{\sigma} \neq 0$.

First, we recall that $J_{d}(X) \simeq H^{1}\left(X, \mathcal{T}_{X}\right)$. Indeed, the normal bundle sequence

$$
\left.0 \rightarrow \mathcal{T}_{X} \rightarrow \mathcal{T}_{\mathbb{P}}\right|_{X} \rightarrow \mathcal{O}_{X}(d) \longrightarrow 0
$$

combined with the restriction of the Euler sequence shows that

$$
J_{d}(X) \simeq \operatorname{Coker}\left(H^{0}\left(X, \mathcal{O}_{X}(1)^{\oplus n+2}\right) \stackrel{\left(\partial_{i} f\right)}{\longrightarrow} H^{0}\left(X, \mathcal{O}_{X}(d)\right)\right) \simeq H^{1}\left(X, \mathcal{T}_{X}\right) .
$$

Moreover, the discussion in Sections 2.4 and 2.5 (cf. Lemma 2.19) shows that the map

$$
H^{1}\left(X, \mathcal{T}_{X}\right) \simeq J_{d}(X) \stackrel{\pi_{d}}{\longrightarrow} L_{d}(X)=\operatorname{Hom}\left(P_{0}, P_{d}\right) \simeq \mathrm{HH}^{2}\left(\mathcal{A}_{X}\right)
$$

can be described as the composition of the standard injection $H^{1}\left(X, \mathcal{T}_{X}\right) \hookrightarrow \mathrm{HH}^{2}(X)$ with the projection $\mathrm{HH}^{2}(X) \longrightarrow \mathrm{HH}^{2}\left(\mathcal{A}_{X}\right)$; see [Kuz09]. The latter is obtained by applying left projection: $j^{*}: \mathrm{HH}^{2}(X)=\operatorname{Hom}\left(\mathcal{O}_{\Delta}, \mathcal{O}_{\Delta}[2]\right) \rightarrow \operatorname{Hom}\left(j^{*} \mathcal{O}_{\Delta}, j^{*} \mathcal{O}_{\Delta}[2]\right)=\operatorname{Hom}\left(P_{0}, P_{0}[2]\right)=\mathrm{HH}^{2}\left(\mathcal{A}_{X}\right)$. In particular, there exists a commutative diagram

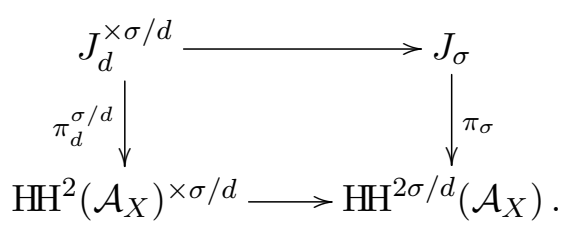

On the other hand, for the Calabi-Yau category $\mathcal{A}_{X}$, one knows by [Kuz18, Proposition 5.3] that there are isomorphisms $\mathrm{HH}^{k}\left(\mathcal{A}_{X}\right) \simeq \mathrm{HH}_{k-\sigma / d}\left(\mathcal{A}_{X}\right)$ compatible with the multiplication on 


\section{HOCHSCHILD COHOMOLOGY VERSUS THE JACOBIAN RING}

$\mathrm{HH}^{*}\left(\mathcal{A}_{X}\right)$ and the $\mathrm{HH}^{*}\left(\mathcal{A}_{X}\right)$-module structure of $\mathrm{HH}_{*}\left(\mathcal{A}_{X}\right)$. So, (3.5) can be completed by the commutative diagram

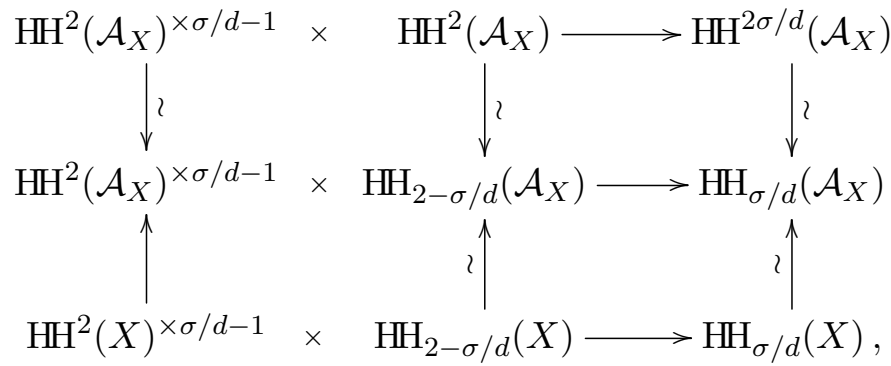

where the lower vertical arrows between the Hochschild homology groups are indeed isomorphisms as long as we assume $\sigma / d>2$; see [Kuz09]. To conclude, use the isomorphism between $\left(\mathrm{HH}^{*}(X), \mathrm{HH}_{*}(X)\right)$ and $\left(H T^{*}(X):=\bigoplus_{r+s=*} H^{r}\left(X \bigwedge^{s} \mathcal{T}_{X}\right), H \Omega^{*}(X):=\bigoplus_{q-p=*} H^{p, q}(X)\right)$, see [Căl05], and the fact that $H^{1}\left(X, \mathcal{T}_{X}\right)^{\times \sigma / d-1} \times H \Omega^{2-\sigma / d}(X) \rightarrow H \Omega^{\sigma / d}(X)$ is non-trivial due to a result of Griffiths; cf. [Don83, Theorem 2.2]. Altogether, this proves $\pi_{\sigma} \neq 0$.

Let us now come to the case $\sigma / d=2$, which is the case of cubic fourfolds and for which the proof is more direct. Here, we know that

$$
J_{3}(X) \simeq H^{1}\left(X, \mathcal{T}_{X}\right) \rightarrow \mathrm{HH}^{2}\left(\mathcal{A}_{X}\right)=L_{3}(X)
$$

is injective with image a subspace of codimension two of the 22-dimensional $\operatorname{HH}^{2}\left(\mathcal{A}_{X}\right)$. If $\pi_{6}$ were trivial, then the non-degenerate pairing $\mathrm{HH}^{2}\left(\mathcal{A}_{X}\right) \times \mathrm{HH}^{2}\left(\mathcal{A}_{X}\right) \rightarrow \mathrm{HH}^{4}\left(\mathcal{A}_{X}\right) \simeq k$ would be trivial on a subspace whose dimension exceeds the maximal dimension of an isotropic subspace. This is the contradiction which allows us to conclude that $\pi_{6} \neq 0$ and hence all $\pi_{\ell}$ are indeed injective.

3.3. We now introduce the version of Hochschild cohomology of $\mathcal{A}_{X}$ of a smooth hypersurface $X \subset \mathbb{P}^{n+1}$ that is appropriate for our purpose.

Definition 3.6. The Hochschild cohomology of the pair $\left(\mathcal{A}_{X},(1)\right)$ is the graded subalgebra

$$
\mathrm{HH}^{*}\left(\mathcal{A}_{X},(1)\right) \subset L(X)
$$

generated by $L_{1}(X)$.

The next result is Theorem 1.1.

COROLlary 3.7. There exists a surjection of graded rings

$$
J(X) \longrightarrow \mathrm{HH}^{*}\left(\mathcal{A}_{X},(1)\right),
$$

which is an isomorphism if $n+2$ divisible by $d<n+2$.

Proof. The first assertion follows from the definition of $\mathrm{HH}^{*}\left(\mathcal{A}_{X},(1)\right)$, Lemma 3.1, and Proposition 3.3. The second part is an immediate consequence of Proposition 3.5.

Remark 3.8. Note that the natural gradings of $\mathrm{HH}^{*}\left(\mathcal{A}_{X}\right)$ and $\mathrm{HH}^{*}\left(\mathcal{A}_{X},(1)\right)$ are not compatible, as, for example, $\mathrm{HH}^{2 \ell}\left(\mathcal{A}_{X}\right)$ is mapped into $L_{d \ell}(X)$. Also, the projections $\pi_{\ell}: J_{\ell}(X) \rightarrow L_{\ell}(X)$ are in general not surjective; that is, $\operatorname{HH}^{*}\left(\mathcal{A}_{X},(1)\right) \subset L(X)$ is a proper subalgebra. For example, for the cubic fourfold, we have $\operatorname{dim} J_{3}(X)=\operatorname{dim} H^{1}\left(X, \mathcal{T}_{X}\right)=20$, whereas $\operatorname{dim} L_{3}(X)=$ $\operatorname{dim} \mathrm{HH}^{2}\left(\mathcal{A}_{X}\right)=22$. 


\section{Huybrechts And J. V. Rennemo}

3.4. Consider two smooth hypersurfaces $X, X^{\prime} \subset \mathbb{P}^{n+1}$ of degree $1<d \leqslant(n+2) / 2$ and their associated categories $\mathcal{A}_{X} \subset \mathrm{D}^{\mathrm{b}}(X)$ and $\mathcal{A}_{X^{\prime}} \subset \mathrm{D}^{\mathrm{b}}\left(X^{\prime}\right)$. We denote the degree-shift functors by (1): $\mathcal{A}_{X} \stackrel{\sim}{\longrightarrow} \mathcal{A}_{X^{\prime}}$ and $(1)^{\prime}: \mathcal{A}_{X^{\prime}} \stackrel{\sim}{\longrightarrow} \mathcal{A}_{X^{\prime}}$ and their natural kernels by $P_{1} \in \mathcal{A}_{X}(-(n+1-d)) \otimes$ $\mathcal{A}_{X}$ and $P_{1}^{\prime} \in \mathcal{A}_{X^{\prime}}(-(n+1-d)) \otimes \mathcal{A}_{X^{\prime}}$; see Example 2.9(ii).

Proposition 3.9. Under the above assumption, let $\Phi=\bar{\Phi}_{P}: \mathcal{A}_{X} \stackrel{\sim}{\rightarrow} \mathcal{A}_{X^{\prime}}$ be a Fourier-Mukai equivalence. Then an isomorphism of the natural Fourier-Mukai kernels of $\Phi \circ(1)$ and $(1)^{\prime} \circ \Phi$,

$$
P_{1} \circ P \simeq P \circ P_{1}^{\prime}
$$

induces an isomorphism of graded algebras

$$
\mathrm{HH}^{*}\left(\mathcal{A}_{X},(1)\right) \simeq \mathrm{HH}^{*}\left(\mathcal{A}_{X^{\prime}},(1)^{\prime}\right) .
$$

Proof. By the definition of a Fourier-Mukai functor, $P \in \mathcal{A}_{X}(-(n+1-d)) \otimes \mathcal{A}_{X^{\prime}}$; see Remark 2.8. Hence, both sides of (3.8) are objects in $\mathcal{A}_{X}(-(n+1-d)) \otimes \mathcal{A}_{X^{\prime}}$. Moreover, successive convolution with $P_{1}$ from the left yields isomorphisms $P_{\ell} \circ P \simeq P \circ P_{\ell}^{\prime}$ for all $\ell \geqslant 0$. Alternatively, $P_{\ell} \circ P$ can be seen as the image of $P_{\ell}$ under the equivalence $\left.(\circ P) \simeq \operatorname{id} \otimes \Phi: \mathcal{A}_{X}(-n+1-d)\right) \otimes \mathcal{A}_{X} \stackrel{\sim}{\rightarrow} \mathcal{A}_{X}(-n+$ $1-d)) \otimes \mathcal{A}_{X^{\prime}}$ and $P \circ P_{\ell}^{\prime}$ as the image of $P_{\ell}^{\prime}$ under the equivalence $(P \circ) \simeq \Psi \otimes \mathrm{id}: \mathcal{A}_{X^{\prime}}(-(n+$ $1-d)) \otimes \mathcal{A}_{X^{\prime}} \stackrel{\sim}{\longrightarrow} \mathcal{A}_{X}(-(n+1-d)) \otimes \mathcal{A}_{X^{\prime}}$. Here, $\Psi: \mathcal{A}_{X^{\prime}}(-(n+1-d)) \stackrel{\sim}{\longrightarrow} \mathcal{A}_{X}(-(n+1-d))$ is the Fourier-Mukai equivalence with kernel in $\mathcal{A}_{X^{\prime}} \otimes \mathcal{A}_{X}(-(n+1-d))$ given by applying the transposition to $P$. The arguments in the geometric case, that is, when $\mathcal{A}_{X}$ is the bounded derived category of a variety, can be easily adapted to show that $\Psi$ is indeed an equivalence.

Hence, the equivalence $(\Psi \nabla \mathrm{id})^{-1} \circ(\mathrm{id} \nabla \Phi): \mathcal{A}_{X}(-(n+1-d)) \otimes \mathcal{A}_{X} \stackrel{\sim}{\longrightarrow} \mathcal{A}_{X^{\prime}}(-(n+1-d)) \otimes \mathcal{A}_{X^{\prime}}$ sends $P_{\ell}$ to $P_{\ell}^{\prime}$ and, therefore, defines isomorphisms $L_{\ell}(X) \simeq L_{\ell}\left(X^{\prime}\right)$, for $\ell \geqslant 0$, compatible with composition. Restricted to the sub-algebras generated by $L_{1}$, this yields the desired isomorphism of graded algebras $\mathrm{HH}^{*}\left(\mathcal{A}_{X},(1)\right) \stackrel{\sim}{\longrightarrow} \mathrm{HH}^{*}\left(\mathcal{A}_{X^{\prime}},(1)^{\prime}\right)$.

As it is expected that Fourier-Mukai kernels $P \in \mathcal{A}_{X}(-(n+1-d)) \otimes \mathcal{A}_{X^{\prime}}$ of FourierMukai equivalences $\Phi=\bar{\Phi}_{P}: \mathcal{A}_{X} \stackrel{\sim}{\longrightarrow} \mathcal{A}_{X^{\prime}}$ are unique, an isomorphism (3.8) should exist whenever $\Phi \circ(1) \simeq(1)^{\prime} \circ \Phi$. This is certainly the case when $\mathcal{A}_{X} \simeq \mathrm{D}^{\mathrm{b}}(S, \alpha)$ for a twisted K3 surface $(S, \alpha)$, due to [CS07, Or197].

Corollary 3.10. Let $X, X^{\prime} \subset \mathbb{P}^{n+1}$ be smooth hypersurfaces of degree $1<d \leqslant(n+2) / 2$ with $d \mid(n+2)$. Then an isomorphism (3.8) of the natural Fourier-Mukai kernels of $\Phi \circ(1)$ and $(1)^{\prime} \circ \Phi$, induces an isomorphism of graded algebras $J(X) \simeq J\left(X^{\prime}\right)$ and, therefore, an isomorphism $X \simeq X^{\prime}$.

Proof. The isomorphism between the Jacobian rings follows from Proposition 3.9 and Corollary 3.7. That the isomorphism between the Jacobian rings implies the existence of an isomorphism $X \simeq X^{\prime}$ is an immediate consequence of the Mather-Yau theorem; see [Don83, Proposition 1.1] or [Voi02, Lemma 18.31]. It may be worth noting that the isomorphism $J(X) \simeq J\left(X^{\prime}\right)$ itself may not lift directly to an automorphism of $k\left[x_{0}, \ldots, x_{n+1}\right]$ identifying the equations of $X$ and $X^{\prime}$.

\section{Hodge theory}

The existence of a Hodge isometry $H^{4}(X, \mathbb{Z})_{\mathrm{pr}} \simeq H^{4}\left(X^{\prime}, \mathbb{Z}\right)_{\mathrm{pr}}$ is shown to yield a Hodge isometry $\widetilde{H}\left(\mathcal{A}_{X}, \mathbb{Z}\right) \simeq \widetilde{H}\left(\mathcal{A}_{X^{\prime}}, \mathbb{Z}\right)$ with additional properties. It will subsequently be lifted to an equivalence 


\section{HOCHSCHILD COHOMOLOGY VERSUS THE JACOBIAN RING}

$\mathcal{A}_{X} \simeq \mathcal{A}_{X^{\prime}}$ that on the level of Hochschild cohomology yields an isomorphism between the Jacobian rings. From now on, we work over $\mathbb{C}$.

4.1. Let us briefly recall the Hodge structure of weight two $\widetilde{H}\left(\mathcal{A}_{X}, \mathbb{Z}\right)$ of the $\mathrm{K} 3$ category $\mathcal{A}_{X} \subset \mathrm{D}^{\mathrm{b}}(X)$ associated with a smooth cubic $X \subset \mathbb{P}^{5}$, as defined by Addington and Thomas in $\left[\right.$ AT14]. As a lattice, $\widetilde{H}\left(\mathcal{A}_{X}, \mathbb{Z}\right)$ is the orthogonal complement of $\left\langle\left[\mathcal{O}_{X}\right],\left[\mathcal{O}_{X}(1)\right],\left[\mathcal{O}_{X}(2)\right]\right\rangle \subset$ $K_{\text {top }}(X)$ with the quadratic form given by the Mukai pairing. The Hodge structure is determined by $\widetilde{H}^{2,0}\left(\mathcal{A}_{X}\right)$ defined as the pull-back of $H^{3,1}(X)$ via the Mukai vector. Furthermore, there exists a natural primitive inclusion (see [AT14, Proposition 2.3])

$$
\iota_{X}: H^{4}(X, \mathbb{Z})_{\operatorname{pr}} \hookrightarrow \widetilde{H}\left(\mathcal{A}_{X}, \mathbb{Z}\right),
$$

which is compatible up to sign with the intersection form on $H^{4}(X, \mathbb{Z})_{\text {pr }}$ and the Mukai pairing on $\widetilde{H}\left(\mathcal{A}_{X}, \mathbb{Z}\right)$. Moreover, $\iota_{X}$ respects the Hodge structures (up to Tate twist); that is, it restricts to an isomorphism $H^{3,1}(X) \stackrel{\sim}{\longrightarrow} \widetilde{H}^{2,0}\left(\mathcal{A}_{X}, \mathbb{Z}\right)$. The orthogonal complement of the inclusion is the primitive sublattice spanned by $\lambda_{j}=\left[i^{*} \mathcal{O}(j)\right]$, for $j=1$, 2 , where as before, $i^{*}: \mathrm{D}^{\mathrm{b}}(X) \rightarrow \mathcal{A}_{X}$ is the left adjoint of the natural inclusion $i_{*}: \mathcal{A}_{X} \hookrightarrow \mathrm{D}^{\mathrm{b}}(X)$. The choice of the generators induces an isometry $\operatorname{Im}\left(\iota_{X}\right)^{\perp} \simeq A_{2}$, which we shall tacitly fix throughout. The induced inclusion

$$
H^{4}(X, \mathbb{Z})_{\operatorname{pr}} \oplus A_{2} \hookrightarrow \widetilde{H}\left(\mathcal{A}_{X}, \mathbb{Z}\right)
$$

is of index three, cf. [Huy16, Section 14.0.2], and its quotient $H_{X}:=\widetilde{H}\left(\mathcal{A}_{X}, \mathbb{Z}\right) /\left(H^{4}(X, \mathbb{Z})_{\operatorname{pr}} \oplus A_{2}\right)$ can be viewed naturally as a subgroup of the discriminant group

$$
A_{H^{4}(X, \mathbb{Z})_{\mathrm{pr}} \oplus A_{2}} \simeq A_{H^{4}(X, \mathbb{Z})_{\mathrm{pr}}} \oplus A_{A_{2}} \simeq \mathbb{Z} / 3 \mathbb{Z} \oplus \mathbb{Z} / 3 \mathbb{Z} .
$$

The two projections from $H_{X}$ yield an isomorphism

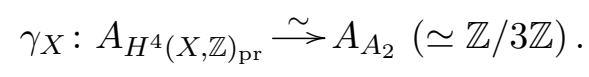

Also note that $H^{4}(X, \mathbb{Z})$ pr with the reversed sign has two positive directions which are naturally oriented by taking the real and imaginary parts of any generator of $H^{3,1}(X)$ (or, equivalently, of $\widetilde{H}^{2,0}\left(\mathcal{A}_{X}\right)$ via $\left.\iota_{X}\right)$. Clearly, by picking the base $\lambda_{1}, \lambda_{2}$ of the positive-definite orthogonal complement $\operatorname{Im}\left(\iota_{X}\right)^{\perp} \simeq A_{2}$, one also gives this part a natural orientation. Put together, the four positive directions of $\widetilde{H}\left(\mathcal{A}_{X}, \mathbb{Z}\right)$ are endowed with a natural orientation.

The orthogonal group of $A_{2}$ is known to be $\mathrm{O}\left(A_{2}\right) \simeq \mathfrak{S}_{3} \times \mathbb{Z} / 2 \mathbb{Z}$. Here, the second factor acts by a global sign change, whereas the first one is the Weyl group acting by permutation of the unit vectors $e_{i}$, where we have an embedding $A_{2} \longleftrightarrow \mathbb{R}^{3}$ via $\lambda_{1} \longmapsto e_{1}-e_{2}$ and $\lambda_{2} \longmapsto e_{2}-e_{3}$. Note that $\mathfrak{S}_{3}$ can also be described as the kernel of the restriction $\mathrm{O}\left(A_{2}\right) \rightarrow \mathrm{O}\left(A_{A_{2}}\right) \simeq \mathrm{O}(\mathbb{Z} / 3 \mathbb{Z}) \simeq \mathbb{Z} / 2 \mathbb{Z}$. Moreover, $g \in \mathrm{O}\left(A_{2}\right)$ preserves the natural orientation of the lattice $A_{2}$ if and only if it is contained in $\mathfrak{A}_{3} \times \mathbb{Z} / 2 \mathbb{Z}$, which still surjects onto $\mathrm{O}\left(A_{A_{2}}\right)$. A generator of $\mathfrak{A}_{3}$ is described by the cyclic permutation of $\lambda_{1}, \lambda_{2},-\lambda_{1}-\lambda_{2}$; cf. [Huy17, Remark 2.1].

Remark 4.1. The category $\mathcal{A}_{X}$ is equipped with the natural auto-equivalence (1): $\mathcal{A}_{X} \stackrel{\sim}{\longrightarrow} \mathcal{A}_{X}$ which is of Fourier-Mukai type; see Example 2.9(ii). The induced action

$$
(1)^{H}: \widetilde{H}\left(\mathcal{A}_{X}, \mathbb{Z}\right) \stackrel{\sim}{\longrightarrow} \widetilde{H}\left(\mathcal{A}_{X}, \mathbb{Z}\right)
$$

is the identity on $H^{4}(X, \mathbb{Z})_{\mathrm{pr}}$ and cyclically permutes $\lambda_{1}, \lambda_{2},-\lambda_{1}-\lambda_{2}$ in the orthogonal complement $A_{2}$; cf. [Huy17, Proposition 3.12]. 


\section{Huybrechts And J. V. Rennemo}

4.2. Any Hodge isometry $\varphi: H^{4}(X, \mathbb{Z}) \stackrel{\sim}{\longrightarrow} H^{4}\left(X^{\prime}, \mathbb{Z}\right)$ mapping $h_{X}$ to $h_{X^{\prime}}$ induces a Hodge isometry $\varphi: H^{4}(X, \mathbb{Z})_{\mathrm{pr}} \stackrel{\sim}{\longrightarrow} H^{4}\left(X^{\prime}, \mathbb{Z}\right)_{\mathrm{pr}}$. Conversely, adapting [Nik80, Corollary 1.5.2] to the present context, one shows that any Hodge isometry $H^{4}(X, \mathbb{Z})_{\mathrm{pr}} \stackrel{\sim}{\rightarrow} H^{4}\left(X^{\prime}, \mathbb{Z}\right)_{\text {pr }}$ extends to a Hodge isometry $H^{4}(X, \mathbb{Z}) \stackrel{\sim}{\longrightarrow} H^{4}\left(X^{\prime}, \mathbb{Z}\right)$ mapping $h_{X}$ to $\pm h_{X^{\prime}}$. (Use the natural isomorphism between the discriminant groups $A_{H^{4}(X, \mathbb{Z})_{\mathrm{pr}}} \simeq A_{A_{2}} \simeq \mathbb{Z} / 3 \mathbb{Z}$.)

For the following, both primitive cohomologies are considered with their natural inclusions $\iota_{X}: H^{4}(X, \mathbb{Z})_{\mathrm{pr}} \hookrightarrow \widetilde{H}\left(\mathcal{A}_{X}, \mathbb{Z}\right)$ and $\iota_{X^{\prime}}: H^{4}\left(X^{\prime}, \mathbb{Z}\right)_{\mathrm{pr}} \hookrightarrow \widetilde{H}\left(\mathcal{A}_{X^{\prime}}, \mathbb{Z}\right)$.

Proposition 4.2. Any Hodge isometry $\varphi: H^{4}(X, \mathbb{Z})_{\mathrm{pr}} \stackrel{\sim}{\sim} H^{4}\left(X^{\prime}, \mathbb{Z}\right)_{\mathrm{pr}}$ extends to an orientationpreserving Hodge isometry $\tilde{\varphi}: \widetilde{H}\left(\mathcal{A}_{X}, \mathbb{Z}\right) \stackrel{\sim}{\longrightarrow} \widetilde{H}\left(\mathcal{A}_{X^{\prime}}, \mathbb{Z}\right)$ that commutes with $(1)^{H}$ in $(4.2)$.

Proof. The key is Nikulin's classical result [Nik80, Corollary 1.5.2], see also [Huy16, Chapter 14], showing that $\varphi$ can be extended to an isometry that restricts to a given $g \in \mathrm{O}\left(A_{2}\right)$ between the orthogonal complements (which we have identified with $A_{2}$ ) if and only if $\bar{g} \circ \gamma_{X}=\gamma_{X^{\prime}} \circ$

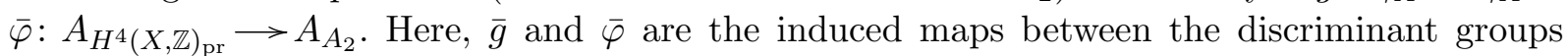
and $\gamma_{X}, \gamma_{X^{\prime}}$ are as in (4.1).

Thus, any lift $g \in \mathrm{O}\left(A_{2}\right)$ of $\gamma_{X^{\prime}} \circ \bar{\varphi} \circ \gamma_{X}^{-1} \in \mathrm{O}\left(A_{A_{2}}\right)$ defines an extension of the Hodge isometry $\varphi$ to an isometry $\tilde{\varphi}: \widetilde{H}\left(\mathcal{A}_{X}, \mathbb{Z}\right) \stackrel{\sim}{\longrightarrow} \widetilde{H}\left(\mathcal{A}_{X^{\prime}}, \mathbb{Z}\right)$ which is then automatically compatible with the Hodge structures. Moreover, there always exists a lift $g \in \mathfrak{A}_{3} \times \mathbb{Z} / 2 \mathbb{Z}$ of $\gamma_{X^{\prime}} \circ \bar{\varphi} \circ \gamma_{X}^{-1}$ such that $\tilde{\varphi}$ not only is a Hodge isometry but also preserves the natural orientation of the four positive directions. Any such $g$ automatically commutes with the cyclic permutation of $\lambda_{1}, \lambda_{2},-\lambda_{1}-\lambda_{2}$, and, therefore, the induced $\tilde{\varphi}$ commutes with $(1)^{H}$.

\section{Deformation theory}

In this section, we show how to combine Corollary 3.10 with results from [Huy17] to prove the global Torelli theorem first for general and then for all cubics.

5.1. We start by recalling [Huy17, Theorem 1.2], which describes the group of auto-equivalences of $\mathcal{A}_{X}$ for a very general cubic. Here, a cubic $X \in|\mathcal{O}(3)|$ is very general if it is contained in the complement of a countable union of proper closed subsets of $|\mathcal{O}(3)|$. The proof in [Huy17] does not depend on the global Torelli theorem for cubic fourfolds, but makes use of results on $\operatorname{Aut}\left(\mathrm{D}^{b}(S, \alpha)\right)$ for twisted K3 surfaces $(S, \alpha)$ without spherical objects; see [HMS08].

Proposition 5.1. Let $X \subset \mathbb{P}^{5}$ be a very general smooth cubic. Then the group $\operatorname{Aut}\left(\mathcal{A}_{X}\right)$ of Fourier-Mukai auto-equivalences $\Phi: \mathcal{A}_{X} \stackrel{\sim}{\longrightarrow} \mathcal{A}_{X}$ (see Definition 2.7) is an infinite cyclic group containing $\mathbb{Z} \cdot[1]$ as an index three subgroup.

The degree-shift functor $(1) \in \operatorname{Aut}\left(\mathcal{A}_{X}\right)$ is symplectic; that is, it acts trivially on the transcendental part (which for a general cubic is $H^{4}(X, \mathbb{Z})_{\mathrm{pr}} \simeq \operatorname{Im}\left(\iota_{X}\right)$ ) and generates the quotient $\operatorname{Aut}_{s}\left(\mathcal{A}_{X}\right) / \mathbb{Z} \cdot[2]$.

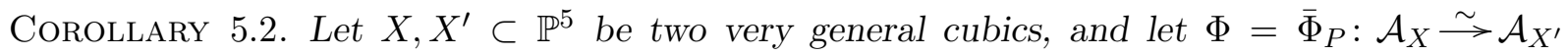
be a Fourier-Mukai equivalence for which the induced action $\Phi^{H}: \widetilde{H}\left(\mathcal{A}_{X}, \mathbb{Z}\right) \stackrel{\sim}{\longrightarrow} \widetilde{H}\left(\mathcal{A}_{X^{\prime}}, \mathbb{Z}\right)$ commutes with the action of the degree shift $(1)^{H}$ on $\widetilde{H}(\mathcal{A}, \mathbb{Z})$. Then $\Phi$ commutes with the degree-shift functor on both sides, that is, $\Phi \circ(1) \simeq(1)^{\prime} \circ \Phi$. 


\section{HOCHSCHILD COHOMOLOGY VERSUS THE JACOBIAN RING}

Proof. Indeed, under the assumption, $\Psi:=\Phi^{-1} \circ(1)^{\prime} \circ \Phi \circ(-1) \in \operatorname{Aut}\left(\mathcal{A}_{X}\right)$ acts trivially on $\widetilde{H}^{1,1}\left(\mathcal{A}_{X}, \mathbb{Z}\right) \simeq A_{2}$ and hence $\Psi \simeq[k]$. However, as $[2] \simeq(3)$ on both sides, the relation $(1)^{\prime} \circ \Phi \simeq \Phi \circ(1) \circ[k]$ automatically implies $k=0$.

If Fourier-Mukai kernels of Fourier-Mukai equivalences $\mathcal{A}_{X} \stackrel{\sim}{\longrightarrow} \mathcal{A}_{X^{\prime}}$ are unique, then $\Phi \circ(1) \simeq$ $(1)^{\prime} \circ \Phi$ would immediately yield (3.8) and hence $X \simeq X^{\prime}$ by Corollary 3.10.

Remark 5.3. In fact, as was stated in [Huy17, Theorem 1.5], two very general cubics $X$ and $X^{\prime}$ are isomorphic if and only if there exists a Fourier-Mukai equivalence $\mathcal{A}_{X} \simeq \mathcal{A}_{X^{\prime}}$. However, the proof given in [Huy17] relies on the global Torelli theorem. Indeed, if $X$ and $X^{\prime}$ are very general, then $\widetilde{H}^{1,1}\left(\mathcal{A}_{X}, \mathbb{Z}\right) \simeq \widetilde{H}^{1,1}\left(\mathcal{A}_{X^{\prime}}, \mathbb{Z}\right)$ is just an isometry of $A_{2}$ and the Hodge isometry of its orthogonal complement can therefore be read as a Hodge isometry $H^{4}(X, \mathbb{Z})_{\mathrm{pr}} \simeq H^{4}\left(X^{\prime}, \mathbb{Z}\right)_{\mathrm{pr}}$.

Remark 5.4. Note that all that was needed in the proof of Corollary 5.2 was that the kernel of $\operatorname{Aut}_{s}\left(\mathcal{A}_{X}\right) \rightarrow \operatorname{Aut}\left(\widetilde{H}\left(\mathcal{A}_{X}, \mathbb{Z}\right)\right)$ is $\mathbb{Z} \cdot[2]$. This is true for very general cubics but also for cubics $X$ for which there exists a twisted K3 surface $(S, \alpha)$ with $\mathcal{A}_{X} \simeq \mathrm{D}^{\mathrm{b}}(S, \alpha)$ and without any $(-2)$ classes in $N(S, \alpha) \simeq \widetilde{H}^{1,1}\left(\mathcal{A}_{X}, \mathbb{Z}\right)$; see [HMS08]. Moreover, in the latter case, $\Phi \circ(1) \simeq(1)^{\prime} \circ \Phi$ implies $P_{\ell} \circ P \simeq P \circ P_{\ell}^{\prime}$, for $\ell \geqslant 1$, due to the uniqueness of Fourier-Mukai kernel for the category of twisted coherent sheaves [CS07].

5.2. Proof of Theorem 1.3. Let $\varphi: H^{4}(X, \mathbb{Z})_{\mathrm{pr}} \stackrel{\sim}{\longrightarrow} H^{4}\left(X^{\prime}, \mathbb{Z}\right)_{\mathrm{pr}}$ be a Hodge isometry. According to Proposition 4.2, the map $\varphi$ can be extended to an orientation-preserving Hodge isometry $\tilde{\varphi}: \widetilde{H}\left(\mathcal{A}_{X}, \mathbb{Z}\right) \stackrel{\sim}{\longrightarrow} \widetilde{H}\left(\mathcal{A}_{X^{\prime}}, \mathbb{Z}\right)$ that commutes with the action of the degree shift $(1)^{H}$ on the two sides, which corresponds to a cyclic permutation of $\lambda_{1}, \lambda_{2},-\lambda_{1}-\lambda_{2}$.

Due to the local Torelli theorem, $\varphi$ can be naturally extended to Hodge isometries

$$
\varphi_{t}: H^{4}\left(X_{t}, \mathbb{Z}\right)_{\mathrm{pr}} \stackrel{\sim}{\rightarrow} H^{4}\left(X_{t}^{\prime}, \mathbb{Z}\right)_{\mathrm{pr}}
$$

for all local deformations $X_{t}$ and $X_{t}^{\prime}$. More precisely, there exists an identification $\operatorname{Def}(X) \simeq$ $\operatorname{Def}\left(X^{\prime}\right)$ between the bases of the universal deformation spaces (think of them as open sets of the period domain) such that parallel transport induces (5.1). Simultaneously, the $\varphi_{t}$ can be extended to orientation-preserving Hodge isometries $\tilde{\varphi} t: \widetilde{H}\left(\mathcal{A}_{X_{t}}, \mathbb{Z}\right) \stackrel{\sim}{\longrightarrow} \widetilde{H}\left(\mathcal{A}_{X_{t}^{\prime}}, \mathbb{Z}\right)$ commuting with the degree shift.

The set $D^{\prime}$ of points $t \in \operatorname{Def}(X) \simeq \operatorname{Def}\left(X^{\prime}\right)$ for which there exists a twisted K3 surface $\left(S_{t}, \alpha_{t}\right)$ without spherical objects and orientation-preserving Hodge isometries

$$
\tilde{\varphi}_{t}: \widetilde{H}\left(\mathcal{A}_{X_{t}}, \mathbb{Z}\right) \stackrel{\sim}{\longrightarrow} \widetilde{H}\left(S_{t}, \alpha_{t}, \mathbb{Z}\right) \stackrel{\sim}{\longrightarrow} \widetilde{H}\left(\mathcal{A}_{X_{t}^{\prime}}, \mathbb{Z}\right)
$$

is dense (more precisely, a countable union of hypersurfaces, which, using their Hodge-theoretic description, can be seen to be also analytically dense); see [Huy17, Corollary 2.16]. Moreover, due to [Huy17, Theorem 1.4], which is the twisted version of [AT14, Theorem 1.1], we may assume that the Hodge isometries (5.2) can be lifted to Fourier-Mukai equivalences

$$
\Phi_{t}=\bar{\Phi}_{P_{t}}: \mathcal{A}_{X_{t}} \stackrel{\sim}{\longrightarrow} \mathrm{D}^{\mathrm{b}}\left(S_{t} \cdot \alpha_{t}\right) \stackrel{\sim}{\longrightarrow} \mathcal{A}_{X_{t}^{\prime}},
$$

where $P_{t} \in \mathcal{A}_{X_{t}}(-2) \otimes \mathcal{A}_{X_{t}^{\prime}}$. Note that, at this point, one implicitly uses the derived global Torelli theorem for K3 surfaces, which ultimately relies on the classical global Torelli theorem for K3 surfaces. For example, it is used in [AT14, Proposition 5.1] and, more generally, in the description of the image of $\operatorname{Aut}\left(\mathrm{D}^{\mathrm{b}}(S, \alpha)\right) \rightarrow \operatorname{Aut}(\widetilde{H}(S, \alpha, \mathbb{Z}))$.

Remark 5.4 applies to $t \in D^{\prime}$ and yields $P_{\ell t} \circ P_{t} \simeq P_{t} \circ P_{\ell t}^{\prime}$, where $P_{\ell t}$ and $P_{\ell t}^{\prime}$ are the natural kernels for the degree-shift functor $(\ell)$ on $\mathcal{A}_{X_{t}}$ and $\mathcal{A}_{X_{t}^{\prime}}$, respectively. Corollary 3.10 then yields 


\section{Huybrechts and J. V. Rennemo}

$X_{t} \simeq X_{t}^{\prime}$ for all $t$ in the dense set $D^{\prime}$ and, as the moduli space of cubics is separated, this shows that $X \simeq X^{\prime}$. This concludes the proof of Theorem 1.3.

Remark 5.5. Note that the arguments in [AT14, Huy17] rely on the fact that all cubics $X \in \mathcal{C}_{8}$ contain a plane. This is a result due to Voisin; see [Voi86, Section 3]. It is used to prove the global Torelli theorem first for these cubics before extending it to all.

In the deformation theory in [AT14, Section 5.2], the authors use the global Torelli theorem to construct a universal family over a finite cover of the moduli space. This can be completely avoided by working with the Hilbert scheme or proved by standard algebraic methods. So, in particular, our arguments to prove the global Torelli theorem are at least not circular.

\section{Further comments}

Although matrix factorizations have not been used in any of the proofs above, some of the arguments are clearly motivated by thinking in terms of $\operatorname{MF}(f, \mathbb{Z})$. We briefly recall the interpretation of $\mathcal{A}_{X}$ as the category of graded matrix factorizations and explain how to view some of the techniques in this light.

The category $\mathcal{A}_{X}$ of a smooth hypersurface $X \subset \mathbb{P}^{n+1}$ can also be described as a category of graded matrix factorizations. More precisely, there exists an exact linear equivalence

$$
\operatorname{MF}(f, \mathbb{Z}) \simeq \mathcal{A}_{X}
$$

where $f \in R_{d}=k\left[x_{0}, \ldots, x_{n+1}\right]_{d}$ is defining $X$. In fact, Orlov constructs a series of fully faithful embeddings $\Phi_{i}: \operatorname{MF}(f, \mathbb{Z}) \hookrightarrow \mathrm{D}^{\mathrm{b}}(X)$, see [Orl09, Theorem 2.5], and for $i=1$, the image is the subcategory $\mathcal{A}_{X}=\langle\mathcal{O}, \ldots, \mathcal{O}(n+1-d)\rangle^{\perp}={ }^{\perp}\langle\mathcal{O}(d-n-2), \ldots, \mathcal{O}(-1)\rangle$.

The objects of $\operatorname{MF}(f, \mathbb{Z})$ are pairs $(K \stackrel{\alpha}{\longrightarrow} L \stackrel{\beta}{\longrightarrow} K(d))$, where $K$ and $L$ are finitely generated, free, graded $R$-modules and $\alpha, \beta$ are graded $R$-module homomorphisms with $\beta \circ \alpha=$ $f \cdot \mathrm{id}=\alpha(d) \circ \beta$. Recall that $K(\ell)$ for a graded $R$-module $K=\bigoplus K_{i}$ is the graded module with $K(\ell)_{i}=K_{\ell+i}$. Morphisms in $\mathrm{MF}(f, \mathbb{Z})$ consist of homotopy classes of pairs of graded homomorphisms $g: K \longrightarrow K^{\prime}$ and $h: L \longrightarrow L^{\prime}$ with $\alpha^{\prime} \circ g=h \circ \alpha$ and $\beta^{\prime} \circ h=g(d) \circ \beta$, and the shift functor making $\operatorname{MF}(f, \mathbb{Z})$ into a triangulated category is given by

$$
(K \stackrel{\alpha}{\longrightarrow} L \stackrel{\beta}{\longrightarrow} K(d))[1]:=(L \stackrel{-\beta}{\longrightarrow} K(d) \stackrel{-\alpha}{\longrightarrow} L(d)) .
$$

The degree-shift functor for $\operatorname{MF}(f, \mathbb{Z})$ is by definition the auto-equivalence given by

$$
\begin{aligned}
(1): \operatorname{MF}(f, \mathbb{Z}) & \stackrel{\sim}{\longrightarrow} \operatorname{MF}(f, \mathbb{Z}), \\
(K \stackrel{\alpha}{\longrightarrow} L \stackrel{\beta}{\longrightarrow} K(d)) & \mapsto(K(1) \stackrel{\alpha(1)}{\longrightarrow} L(1) \stackrel{\beta(1)}{\longrightarrow} K(d+1)),
\end{aligned}
$$

which obviously satisfies $(d) \simeq[2]$; cf. Corollary 2.16 .

Remark 6.1. According to [BFK12, Proposition 5.8], under the fixed equivalence $\operatorname{MF}(f, \mathbb{Z}) \simeq \mathcal{A}_{X}$ (which corresponds to $\Phi_{1}$ in [Orl09, Theorem 2.5]), the degree-shift functor $(1)$ on $\mathrm{MF}(f, \mathbb{Z})$ is isomorphic to the auto-equivalence $i^{*} \circ(\mathcal{O}(1) \otimes())$ of $\mathcal{A}_{X}$, which is the degree-shift functor (1) on $\mathcal{A}_{X}$ as introduced in Example 2.9(ii).

Of course, once this is established, the isomorphism $(d) \simeq[2]$ in Corollary 2.16 is immediate. However, only on the level of functors but not on the level of Fourier-Mukai kernels, which was crucial for our purposes.

Consider the image of a section $s \in H^{0}\left(X, \mathcal{O}_{X}(1)\right)$ under the isomorphism (3.1), and view 


\section{HochSCHILD COHOMOLOGY VERSUS THE JACOBIAN RING}

it as a morphism between Fourier-Mukai kernels $s: P_{0}=j^{*} \mathcal{O}_{\Delta} \longrightarrow P_{1}=j^{*} \mathcal{O}_{\Delta}(1)$. The induced natural transform $s:$ id $\longrightarrow(1)$ between the auto-equivalences id, (1): $\mathcal{A}_{X} \simeq \operatorname{MF}(f, \mathbb{Z}) \stackrel{\sim}{\longrightarrow} \mathcal{A}_{X} \simeq$ $\mathrm{MF}(f, \mathbb{Z})$ can be described in terms of matrix factorizations as given by multiplication by the section $s$, that is,

$$
(K \stackrel{\alpha}{\longrightarrow} L \stackrel{\beta}{\longrightarrow} K(d)) \stackrel{(s, s, s)}{\longrightarrow}(K(1) \stackrel{\alpha(1)}{\longrightarrow} L(1) \stackrel{\beta(1)}{\longrightarrow} K(d+1)) .
$$

A similar description holds for the transforms id $\rightarrow(\ell)$ induced by sections $s \in H^{0}\left(X, \mathcal{O}_{X}(\ell)\right)$.

Remark 6.2. The naive idea behind Proposition 3.3 is the chain rule. Indeed, for $s=\partial_{i} f \in$ $H^{0}\left(X, \mathcal{O}_{X}(d-1)\right)$, the map (6.1) is homotopic to zero; just use the chain rule $\partial_{i} f=\partial_{i}(\beta \circ \alpha)=$ $\partial_{i} \alpha \circ \beta+\alpha \circ \partial_{i} \beta$ to see that $\partial_{i} \beta: L \longrightarrow K(d-1)$ and $\partial_{i} \alpha: K \longrightarrow L(-1)$ define a homotopy $\partial_{i} f \sim 0$; see, for example, [Dyc11]. However, Proposition 3.3 cannot be replaced by this easy observation, as the natural map $\operatorname{Hom}\left(P_{0}, P_{\ell}\right) \rightarrow \operatorname{Fun}(\mathrm{id},(\ell))$ is not always injective. ${ }^{5}$

It seems feasible that some of the arguments in this paper can indeed be rephrased in the language of matrix factorizations. However, the transition between the two points of view is often involved; cf. [BFK12]. Already, identifying the two degree-shift functors (1) or verifying that $s \in H^{0}\left(X, \mathcal{O}_{X}(1)\right)$ really yields (6.1) are non-trivial matters. In the end, we decided that staying in the derived context throughout makes the arguments cleaner.

Passing from the formalism of matrix factorizations to the equivalent formalism of general factorizations (see, for instance, [ADS15, BFK14]), we may represent the functors

$$
(i): \mathrm{MF}(f, \mathbb{Z}) \rightarrow \mathrm{MF}(f, \mathbb{Z})
$$

by a natural Fourier-Mukai kernel $Q_{i}$ in (the appropriately graded version of) $\mathrm{MF}(-f \boxplus f)$. We may then define $L_{\mathrm{MF}}(X):=\bigoplus \operatorname{Hom}\left(Q_{0}, Q_{i}\right)$. A more general version of this ring is computed in [BFK14]. Their methods can presumably be used to show that $J(X)$ is isomorphic to the subring of $L_{\mathrm{MF}}(X)$ generated by its degree one part. It is natural to conjecture that $L_{\mathrm{MF}}(X) \simeq L(X)$, but for the reasons listed, proving this is a non-trivial task.

Acknowledgments. DH thanks Toby Dyckerhoff for explanations concerning [Dyc11], Andrey Soldatenkov for his help with the literature, and Alex Perry for helpful discussions on Kuznetsov's work. JR thanks Arend Bayer and John Calabrese for discussions, and, especially, Matt Ballard, who originally suggested to use [Dyc11] and the Mather-Yau theorem to recover a hypersurface from its category $\mathcal{A}_{X}$. We are grateful to the referee for a careful reading of the paper and helpful comments.

\section{REFERENCES}

ADS15 N. Addington, W. Donovan, and E. Segal, The Pfaffian-Grassmannian equivalence revisited, Algebr. Geom. 2 (2015), no. 3, 332-364; doi:10.14231/AG-2015-015.

AT14 N. Addington and R. Thomas, Hodge theory and derived categories of cubic fourfolds, Duke Math. J. 163 (2014), no. 10, 1885-1927; doi:10.1215/00127094-2738639.

BFK12 M. Ballard, D. Favero, and L. Katzarkov, Orlov spectra: bounds and gaps, Invent. Math. 189 (2012), no. 2, 359-430; doi:10.1007/s00222-011-0367-y.

\footnotetext{
${ }^{5}$ Indeed, it can be shown that Fun(id, $\left.[i]\right)=0$ for the bounded derived category $\mathrm{D}^{\mathrm{b}}(Y)$ of any smooth projective variety $Y$ and for $i>\operatorname{dim}(Y)$. On the other hand, $\operatorname{Hom}\left(P_{0}, P_{\sigma}\right) \neq 0$. So, for example, for cubic fourfolds with $\mathcal{A}_{X} \simeq \mathrm{D}^{\mathrm{b}}(S)$, the map $\operatorname{Hom}\left(P_{0}, P_{6}\right) \longrightarrow \operatorname{Fun}(\mathrm{id},(6)) \simeq$ Fun(id, [4] $)=0$ is not injective.
} 


\section{Huybrechts And J. V. Rennemo}

BFK14_, A category of kernels for equivariant factorizations and its implications for Hodge theory, Publ. Math. Inst. Hautes Études Sci. 120 (2014), 1-111; doi:10.1007/s10240-013-0059-9.

BK89 A. Bondal and M. Kapranov, Representable functors, Serre functors, and reconstructions, Math. USSR-Izv. 53 (1989), no. 6, 519-541; doi:10.1070/IM1990v035n03ABEH000716.

BO95 A. Bondal and D. Orlov, Semiorthogonal decomposition for algebraic varieties, 1995, arXiv:alg-geom/9506012.

BR75 D. Burns, Jr. and M. Rapoport, On the Torelli problem for kählerian $K-3$ surfaces, Ann. Sci. École Norm. Sup. (4) 8 (1975), no. 2, 235-273; doi:10.24033/asens.1287.

Căl05 A. Căldăraru, The Mukai pairing. II. The Hochschild-Kostant-Rosenberg isomorphism, Adv. Math. 194 (2005), no. 1, 34-66; doi:10.1016/j.aim.2004.05.012

CG90 D. A. Cox and M. L. Green, Polynomial structures and generic Torelli for projective hypersurfaces, Compos. Math. 73 (1990), no. 2, 121-124; http://www.numdam.org/item?id=CM_1990_ _73_2_121_0.

Cha12 F. Charles, A remark on the Torelli theorem for cubic fourfolds, 2012, arXiv:1209.4509.

CS07 A. Canonaco and P. Stellari, Twisted Fourier-Mukai functors, Adv. Math. 212 (2007), no. 2, 484-503; doi:10.1016/j.aim.2006.10.010..

DG84 R. Donagi and M.L. Green, A new proof of the symmetrizer lemma and a stronger weak Torelli theorem for projective hypersurfaces, J. Differential Geom. 20 (1984), no. 2, 459-461; doi:10.4310/jdg/1214439288.

Don83 R. Donagi, Generic Torelli for projective hypersurfaces, Compos. Math. 50 (1983), no. 2-3, 325353; http://www. numdam.org/item?id=CM_1983__50_2-3_325_0.

Dyc11 T. Dyckerhoff, Compact generators in categories of matrix factorizations, Duke Math. J. 159 (2011), no. 2, 223-274; doi:10.1215/00127094-1415869.

HMS08 D. Huybrechts, E. Macrì, and P. Stellari, Stability conditions for generic K3 categories, Compos. Math. 144 (2008), no. 1, 134-162; doi:10.1112/S0010437X07003065.

HT10 D. Huybrechts and R.P. Thomas, Deformation-obstruction theory for complexes via Atiyah and Kodaira-Spencer classes, Math. Ann. 346 (2010), no. 3, 545-569; doi:10.1007/s00208-009-0397-6.

Huy06 D. Huybrechts, Fourier-Mukai transforms in algebraic geometry, Oxford Math. Monogr. (Oxford Univ. Press, Oxford, 2006); doi:10.1093/acprof :oso/9780199296866.001.000.

Huy12 , A global Torelli theorem for hyperkähler manifolds [after M. Verbitsky], Astérisque (2012), no. 348, Exp. No. 1040, x, 375-403.

Huy16 L Lectures on K3 surfaces, Cambridge Stud. Adv. Math., vol. 158 (Cambridge Univ. Press, Cambridge, 2016); doi:10.1017/СB09781316594193.

Huy17 , The K3 category of a cubic fourfold, Compos. Math. 153 (2017), no. 3, 586-620; doi:10.1112/S0010437X16008137.

Kuz04 A. Kuznetsov, Derived category of a cubic threefold and the variety $V_{14}$, Proc. Steklov Inst. Math. 246 (2004), no. 3, 171-194.

Kuz09 _ Hochschild homology and semiorthogonal decompositions, 2009, arXiv:0904.4330.

Kuz10 Derived categories of cubic fourfolds, in Cohomological and Geometric Approaches to Rationality Problems, Progr. Math., vol. 282, (Birkhäuser Boston, Inc., Boston, MA, 2010), 219243; doi:10.1007/978-0-8176-4934-0_9.

Kuz11_ B Base change for semiorthogonal decompositions, Compos. Math. 147 (2011), no. 3, 852876; doi:10.1112/S0010437X10005166.

Kuz18_, Calabi-Yau and fractional Calabi-Yau categories, J. reine angew. Math., published online on 2 March 2017, doi:10.1515/crelle-2017-0004, to appear in print.

Loo09 E. Looijenga, The period map for cubic fourfolds, Invent. Math. 177 (2009), no. 1, 213-233; doi:10.1007/s00222-009-0178-6. 


\section{HochSCHILD COHOMOLOGY VERSUS THE JACOBIAN RING}

Mar09 N. Markarian, The Atiyah class, Hochschild cohomology and the Riemann-Roch theorem, J. Lond. Math. Soc. 79 (2009), no. 1, 129-143; doi:10.1112/jlms/jdn064.

Mar11 E. Markman, A survey of Torelli and monodromy results for holomorphic-symplectic varieties, in Complex and Differential Geometry, Springer Proc. Math., vol. 8 (Springer, Heidelberg, 2011), 257-322; doi:10.1007/978-3-642-20300-8_15.

MS12 E. Macrì and P. Stellari, Fano varieties of cubic fourfolds containing a plane, Math. Ann. 354 (2012), no. 3, 1147-1176; doi:10.1007/s00208-011-0776-7.

Nik80 V.V. Nikulin, Integer symmetric bilinear forms and some of their geometric applications, Math. USSR-Izv. 14 (1980), no. 1, 103-167; doi:10.1070/IM1980v014n01ABEH001060.

Orl97 D. Orlov, Equivalences of derived categories and K3 surfaces, J. Math. Sci. 84 (1997), no. 5, 1361-1381; doi:10.1007/BF02399195.

Orl09 - Derived categories of coherent sheaves and triangulated categories of singularities, in Algebra, Arithmetic, and Geometry: in Honor of Yu.I. Manin, Vol. II, Progr. Math., vol. 270 (Birkhäuser Boston, Inc., Boston, MA, 2009), 503-531; doi:10.1007/978-0-8176-4747-6_16.

PS71 I. I. Pjateckiı̌-Šapiro and I. R. Šafarevič, Torelli's theorem for algebraic surfaces of type K3, Math. USSR-Izv. 5 (1971), no. 3, 547-588; doi:10.1070/IM1971v005n03ABEH001075.

SGA7 P. Deligne and N. Katz, Groupes de monodromie en géométrie algébrique (SGA 7 II), Lecture Notes in Math., vol. 340, (Springer-Verlag, Berlin - New York, 1973); doi:10.1007/BFb0060505.

Sha80 J. Shah, A complete moduli space for K3 surfaces of degree 2, Ann. of Math. 112 (1980), no. 3, 485-510; doi:10.2307/1971089.

Ver13 M. Verbitsky, Mapping class group and a global Torelli theorem for hyperkähler manifolds (with Appendix A by Eyal Markman), Duke Math. J. 162 (2013), no. 15, 2929-2986; doi:10.1215/00127094-2382680.

Voi86 C. Voisin, Théorème de Torelli pour les cubiques de $\mathbb{P}^{5}$, Invent. Math. 86 (1986), no. 3, 577-601; doi:10.1007/BF01389270.

Voi99 , A generic Torelli theorem for the quintic threefold, New Trends in Algebraic Geometry (Warwick, 1996), London Math. Soc. Lecture Note Ser., vol. 264 (Cambridge Univ. Press, Cambridge, 1999), 425-463; doi:10.1017/СB09780511721540.017.

Voi02 - Théorie de Hodge et géométrie algébrique complexe, Cours Spécialisés, vol. 10 (Soc. Math. France, Paris, 2002); doi:10.1017/CB09780511615344.

Voi08_ Erratum to "Théorème de Torelli pour les cubiques de $\mathbb{P}^{5}$ " [Invent. Math. 86 (1986), no. 3, 577-601], Invent. Math. 172 (2008), no. 2, 455-458; doi:10.1007/s00222-008-0116-z.

Daniel Huybrechts huybrech@math.uni-bonn.de

Mathematisches Institut, Universität Bonn, Endenicher Allee 60, 53115 Bonn, Germany

Jørgen Vold Rennemo jvrennemo@gmail.com

Mathematical Institute, University of Oxford, Woodstock Road, Oxford OX2 6GG, United Kingdom

Current address: Department of Mathematics, University of Oslo, PO Box 1053, Blindern, 0316

Oslo, Norway 